\title{
A MEGACAM SURVEY OF OUTER HALO SATELLITES. VI. THE SPATIALLY RESOLVED STAR- FORMATION HISTORY OF THE CARINA DWARF SPHEROIDAL GALAXY*
}

\author{
Felipe A. Santana ${ }^{1}$, Ricardo R. Muñoz ${ }^{1}$, T. J. L. de Boer ${ }^{2}$, Joshua D. Simon ${ }^{3}$, Marla Geha ${ }^{4}$, Patrick Côté ${ }^{5}$, \\ Andrés E. GuZmán ${ }^{1}$, Peter Stetson ${ }^{5}$, AND S. G. DJorgovski ${ }^{6}$ \\ ${ }^{1}$ Departamento de Astronomía, Universidad de Chile, Camino El Observatorio 1515, Las Condes, Santiago, Chile; fsantana@das.uchile.cl, rmunoz@das.uchile.cl \\ ${ }^{2}$ Institute of Astronomy, University of Cambridge, Madingley Road, Cambridge CB3 OHA UK \\ ${ }^{3}$ Observatories of the Carnegie Institution of Washington, 813 Santa Barbara Street, Pasadena, CA 91101, USA \\ ${ }^{4}$ Astronomy Department, Yale University, New Haven, CT 06520, USA \\ ${ }^{5}$ National Research Council of Canada, Herzberg Astronomy and Astrophysics Program, Victoria, BC, V9E 2E7, Canada \\ ${ }^{6}$ Astronomy Department, California Institute of Technology, Pasadena, CA, 91125, USA \\ Received 2016 April 29; revised 2016 July 8; accepted 2016 July 11; published 2016 September 26
}

\begin{abstract}
We present the spatially resolved star-formation history (SFH) of the Carina dwarf spheroidal galaxy, obtained from deep, wide-field $g$ and $r$ imaging and a metallicity distribution from the literature. Our photometry covers $\sim 2 \mathrm{deg}^{2}$, reaching up to $\sim 10$ times the half-light radius of Carina with a completeness higher than $50 \%$ at $g \sim 24.5$, more than one magnitude fainter than the oldest turnoff. This is the first time a combination of depth and coverage of this quality has been used to derive the SFH of Carina, enabling us to trace its different populations with unprecedented accuracy. We find that Carina's SFH consists of two episodes well separated by a star-formation temporal gap. These episodes occurred at old ( $>10 \mathrm{Gyr})$ and intermediate (2-8 Gyr) ages. Our measurements show that the old episode comprises the majority of the population, accounting for $54 \pm 5 \%$ of the stellar mass within 1.3 times the King tidal radius, while the total stellar mass derived for Carina is $1.60 \pm 0.09 \times 10^{6} M_{\odot}$, and the stellar mass-to-light ratio is $1.8 \pm 0.2$. The SFH derived is consistent with no recent star formation, which hints that the observed blue plume is due to blue stragglers. We conclude that the SFH of Carina evolved independently of the tidal field of the Milky Way, since the frequency and duration of its star-formation events do not correlate with its orbital parameters. This result is supported by the age-metallicity relation observed in Carina and the gradients calculated indicating that outer regions are older and more metal-poor.
\end{abstract}

Key words: galaxies: dwarf - galaxies: evolution - galaxies: stellar content - Hertzsprung-Russell and C-M diagrams - Local Group

\section{INTRODUCTION}

Dwarf galaxies are crucial for understanding galaxy assembly and evolution. They are some of the oldest systems in the universe and inhabit the most numerous type of darkmatter halos in the framework of a Lambda-CDM universe (e.g., Kauffmann et al. 1993). These systems gave origin to larger galaxies like the Milky Way in the early universe via hierarchical merging (e.g., Unavane et al. 1996). The dwarf galaxies in the Local Group are particularly interesting because their proximity allows us to resolve them into individual stars. Thus, it is not surprising that these galaxies have been studied in more detail than any other galaxies (Tolstoy et al. 2009; McConnachie 2012).

Carina is a Local Group dwarf spheroidal (dSph) galaxy located at about $104 \mathrm{kpc}$ from the Sun (Karczmarek et al. 2015), with a half-light radius of $250 \pm 39$ pc (Irwin \& Hatzidimitriou 1995), a dynamical mass within the halflight radius of $M_{\text {dyn }}\left(<r_{\text {half }}\right)=3.4 \pm 1.4 \times 10^{6} M_{\odot}$ (Walker et al. 2009), and an absolute magnitude of $M_{V}=-9.3$ (Mateo 1998). This galaxy is especially important as a constraint on Galactic evolution since it is one of the few Local Group galaxies showing an episodic star-formation history (SFH) (e.g., Weisz et al. 2014b). Furthermore, Carina is

* Based on observations obtained with the MegaCam imager on the Magellan II-Clay telescope at Las Campanas Observatory in the Atacama Region, Chile. This telescope is operated by a consortium consisting of the Carnegie Institution of Washington, Harvard University, MIT, the University of Michigan, and the University of Arizona. the only galaxy where the episodic SFH translates into two clearly distinct subgiant branches (see for example Bono et al. 2010; de Boer et al. 2014). These star-formation episodes may be either related to interactions with the Milky Way or with internal evolution of its gas and stars.

The SFH of local systems like Carina have been studied mainly through the analysis of their color-magnitude diagrams (CMDs). In recent years this has been done using the synthetic CMD method (e.g., de Boer et al. 2014; Weisz et al. 2014b). This technique consists of deriving the history of a stellar system by creating different combinations of synthetic single stellar populations and comparing their properties to the ones of the stars observed. Carina was first thought to be a purely intermediate-age population galaxy, but then RR-Lyraes were discovered in this system, indicating that an old ( $>10 \mathrm{Gyr})$ stellar population was also present in this galaxy (Saha et al. 1986). Multiple main-sequence turnoffs confirmed that Carina had an episodic SFH (Smecker-Hane et al. 1996; Hurley-Keller et al. 1998), which means that there are clearly distinguishable episodes of star-formation activity separated by episodes where practically no stars were formed. Another key feature of Carina's CMD is its narrow red giant branch (RGB), which was at first interpreted as the result of a low metallicity spread (see Rizzi et al. 2003, and references therein). In that work, the authors measured a color spread of the RGB of $\sigma_{\mathrm{V}-\mathrm{I}}=0.021 \pm 0.005$ and derived a metallicity of $[\mathrm{Fe} / \mathrm{H}]=-1.91$ with a spread of $0.12 \mathrm{dex}$, in agreement with early spectroscopic studies of upper RGB stars in Carina (e.g., Armandroff \& Da Costa 1991). More recent spectroscopic 
observations have detected a much larger metallicity spread in this galaxy (Helmi et al. 2006; Koch et al. 2006). The latter study measured a mean metallicity of $[\mathrm{Fe} / \mathrm{H}] \sim-1.4$ and a spread of 0.92 dex. More recently, de Boer et al. (2014) used Koch's metallicity distribution function (MDF) along with CMD information from their deep photometry and derived a self-consistent, complex SFH for Carina, indicating a strong age-metallicity degeneracy. This degeneracy implies that some properties of a given stellar population, like its MDF or its RGB color and width, are equivalent to the ones of a population that is older and more metal-poor. Confirmation or refutation of these results would shed important light on the origin of Carina's photometric and spectroscopic features.

From all these previous works, there is a general agreement that Carina has a well-separated, episodic SFH consisting of at least two episodes producing old and intermediate-age populations. In addition, several studies (Hurley-Keller et al. 1998; Mateo et al. 1998; Monelli et al. 2003) claimed a third episode in Carina, consisting of young $(<1 \mathrm{Gyr})$ stars. However, the exact age and duration of all these potential episodes are still uncertain.

Another important feature found in Carina is the metallicity and age radial gradients of its stellar populations. In Carina's outer regions, the relative prevalence of older and more metalpoor stars increases (Muñoz et al. 2006; Battaglia et al. 2012; de Boer et al. 2014; McMonigal et al. 2014). One of these contributions, Muñoz et al. (2006), presented several observations that were indicative of tidal effects on Carina, a plausible explanation for these population gradients. These pieces of evidence include a component in the density profile that extends well beyond the nominal King limiting radius, a distribution of outer stars that lie preferentially along the major axis, and a velocity dispersion profile that rises well past the limiting radius. These results were then supported by the work of Muñoz et al. (2008) and Battaglia et al. (2012). It is worth mentioning that the proper motion study of Carina by Piatek et al. (2003) results in an orbit that is consistent with the tidal scenario proposed by Muñoz et al. (2008). In their study, Piatek et al. (2003) claimed that this $\mathrm{dSph}$ is currently at apocenter and has an orbital period close to $2 \mathrm{Gyr}$, and its last close passage to the Milky Way occurred $\sim 0.7$ Gyr ago. Tidal effects are interesting in the context of the work by Piatek et al. (2003) and Pasetto et al. (2011), who tested if the star-formation episodes of Carina could be explained as the result of close encounters with the Milky Way. These encounters can promote star formation, for example, by removing angular momentum from the gas and driving it to the central regions (e.g., Larson 2002) or by compression produced by tidal gravitational shocks (e.g., Pasetto et al. 2011). They placed constraints on Carina's orbit but have achieved limited success in explaining Carina's $\mathrm{SFH}$ as a result of tidal shocks or ram pressure.

Another proposed origin for the properties of Carina's SFH is internal evolution. For example, gas depletion or radial migration (El-Badry et al. 2015) might produce the positive age radial gradient (average stellar population age increasing with radius) and the negative metallicity gradient (average metal content decreasing with radius). Additionally, gas heating (see for example Revaz et al. 2009) might explain the temporal gap in star formation.

In summary, we do not have at present a complete scenario explaining the evolution of Carina that is consistent with its
SFH, chemical enrichment, orbital information, and gas dynamics. In this work we use deep and wide photometry along with public metallicities to derive the SFH of Carina. By making use of the Talos routine (de Boer et al. 2012), we take into consideration all the information in the CMD (and not just some key fingerprints) along with the MDF to derive the SFH in a consistent way. The high quality of the photometry, along with the spatial extent of the observations (two square degrees), enables us to make three independent measures of Carina's SFH within different concentric regions. In this way, we can quantify the dependence of the SFH on the distance from the galaxy center by looking at radial gradients.

In Section 2 we present the spectroscopic and photometric data. Section 3 describes the method for deriving the SFH along with the input files used. Section 4 presents the main results for the SFH, which are discussed in Section 5.

\section{DATA}

The observations used in this article are part of a larger survey to obtain deep and wide photometry of all the Milky Way satellites in the outer halo (R. R. Muñoz et al. 2016, in preparation). The main structural parameters of Carina used in this study were derived in that same work by fitting different density profiles to the data and using a maximumlikelihood method. From this work, we took the King tidal radius of Carina $\left(r_{\text {tidal }}=31\right.$. $\left.0 \pm 0.3\right)$, which was estimated as the limiting radius. The other structural parameters used from this study are the central coordinates of Carina $\left(\alpha_{0}=06.41^{\mathrm{m}} .01^{\mathrm{s}} .70, \quad \delta_{0}=-50^{\circ} 57.58^{\prime \prime} 0\right), \quad$ the ellipticity $(\epsilon=0.35 \pm 0.01)$, and the position angle $(\theta=61 \pm 1)$.

\subsection{Photometry}

Images for Carina were obtained using the MegaCam imager on the Magellan II-Clay telescope at Las Campanas Observatory in the Atacama Region, Chile. A total of 16 fields in a $4 \times 4$ configuration were observed, centered on Carina, achieving a total area of $\sim 2 \mathrm{deg}^{2}$. This translates into a full coverage within 1.3 times the tidal radius of Carina and partial coverage from 1.3 to $3.0 \times r_{\text {tidal }}$. For each field observed, we took five dithered exposures in the Clay $g$ and $r$ bands, using a standard dithering pattern from the MegaCam operation options in order to cover the gaps between the chips. The exposure times were $90 \mathrm{~s}$ for the $g$ images and $180 \mathrm{~s}$ for the $r$ images.

The images were first processed to correct for bias levels, variations in the pixel-to-pixel sensitivity, and bad pixels. Point-spread function photometry was then carried out simultaneously in the overlapping images by using the packages DAOPHOT, Allstar, and ALLFRAME (Stetson 1994), as described in Muñoz et al. (2010). From all the sources obtained with our photometry, we selected the ones that are most likely to correspond to stars. To make this distinction, we used the DAOPHOT parameters $C h i$ and sharpness, indicating the significance of the point-spread function fitting and the thickness of the brightness profile, respectively. The sources selected as stars were the ones with magnitude uncertainties smaller than 0.1 in both the $g$ and $r$ bands and satisfying $\mathrm{Chi}<5$ and $-0.4<$ sharp $<0.4$. Finally, we calibrated our photometry by transforming our Clay $g$ and $r$ instrumental magnitudes into the Sloan Digital Sky Survey (York et al. 2000) $g$ and $r$ magnitudes. For this, we 

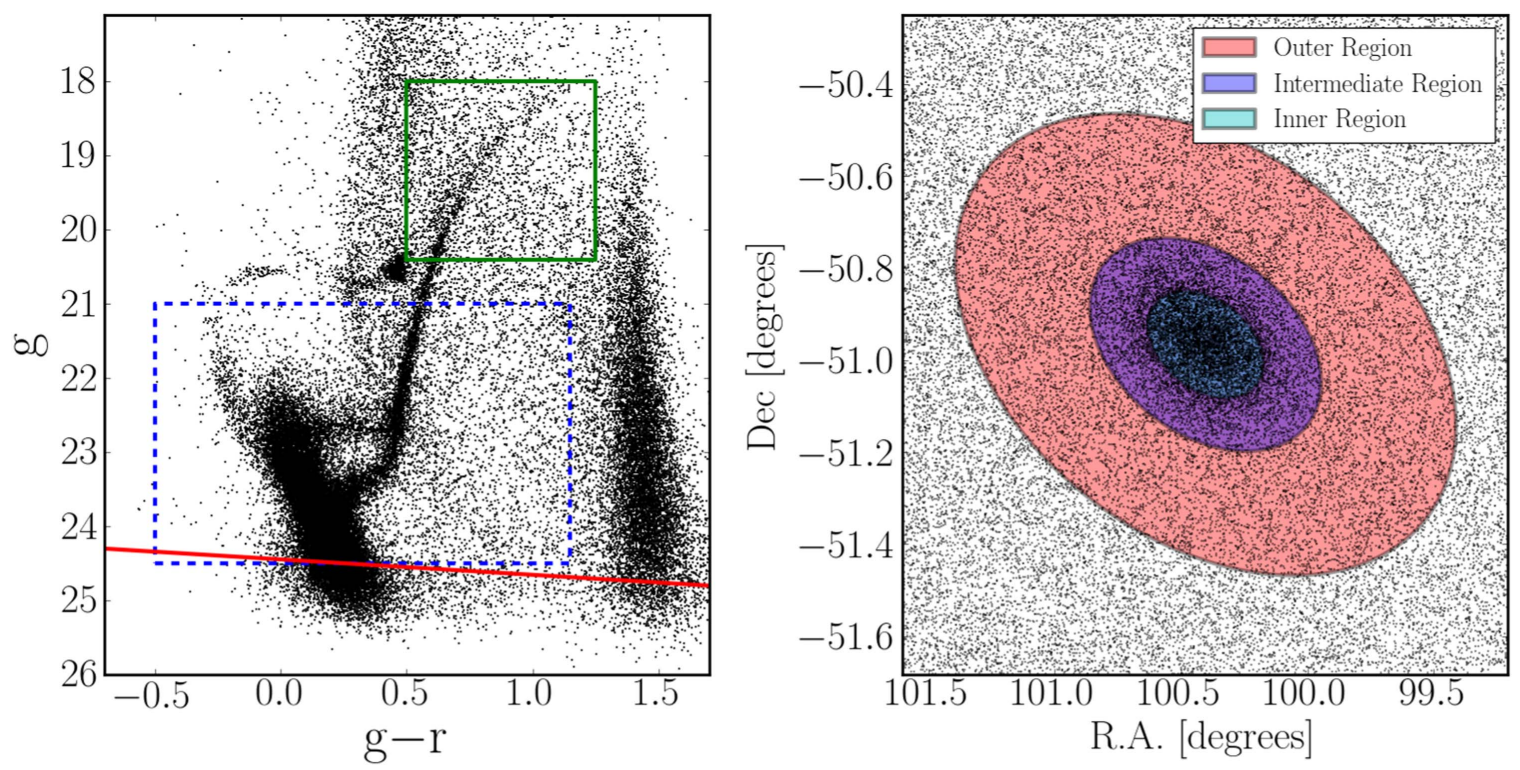

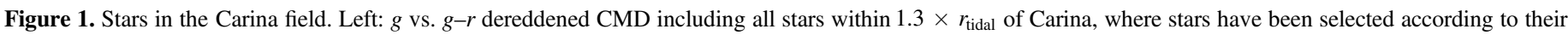

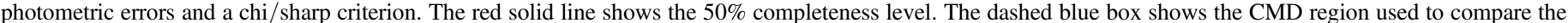

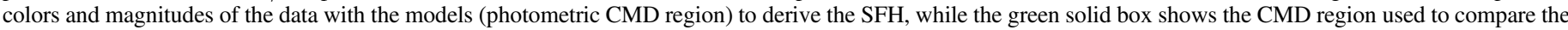

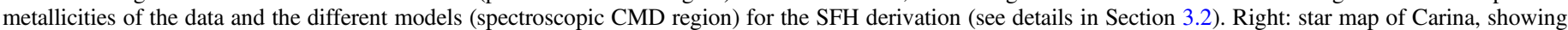

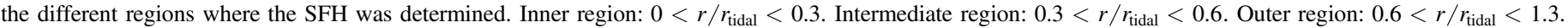

used the photometry from the globular clusters and dwarf galaxies from our photometric catalog of Milky Way satellites that overlap the Sloan footprint. By comparing the magnitudes of the stars in these systems with those in Sloan, we derived the zero points, color, and extinction terms that were then applied to calibrate the magnitudes of the stars in our Carina data. Figure 1 shows the spatial coverage along with the final calibrated CMD of all the sources selected as stars.

\subsection{Spectroscopy}

We used archival, medium-resolution $(R \sim 6500)$ Ca II triplet spectroscopy from Koch et al. (2006). The targets were chosen from the ESO imaging study (Nonino et al. 1999) sample, according to their photometry and astrometry. From that sample, the authors selected the stars across the full width of the RGB ( 0.2 magnitudes in $B-V$ ), from the RGB tip down to 3 mag below the RGB tip. By choosing a wide color range, they ensured that potentially extremely metal-poor or metal-rich giants were included. The complete spectroscopic sample of Koch et al. (2006) included 1257 stars in the region centered on Carina, covering approximately $1 \mathrm{deg}^{2}$ and reaching in all cases a signalto-noise ratio of at least 20 . Then, the members of the Carina galaxy were chosen as the sources with radial velocities consistent with the systematic heliocentric radial velocity of Carina of $\sim 220 \mathrm{~km} \mathrm{~s}^{-1}$. The final catalog of kinematic members of Carina from Koch et al. (2006) contains 437 sources. From this sample, 430 were matched to stars in our photometric data by selecting sources that were closer than 1 arcsecond from a star belonging to our sample. ${ }^{7}$ Both the magnitudes and the positions of the spectroscopic sample were taken from our photometric catalogs to make them directly comparable to the photometric sample. To determine the iron abundances, Koch et al. (2006)

\footnotetext{
7 Given that the typical distances from stars in our photometric sample are at the order of tens of arcseconds even in the highest-density regions, this matching criterion is extremely unlikely to produce matches between observations that do not correspond to the same star.
}

translated the equivalent widths of Ca II using the calibrations of Zinn \& West (1984) and Carretta \& Gratton (1997). However, in this study we used the calibration of Starkenburg et al. (2010) to obtain the $[\mathrm{Fe} / \mathrm{H}]$ values, mainly to avoid saturation at low equivalent widths. Our final sample comprises measurements out to $1.0 \times r_{\text {tidal }}$ of Carina and spans a range in metallicity of $-3.8<[\mathrm{Fe} / \mathrm{H}]<0.0$.

\section{SFH DERIVATION METHOD}

To derive the SFH of Carina, we used the Talos routine (de Boer et al. 2012). In this section we describe its main features, how it was implemented for the case of our Carina data, and the construction of the necessary input files.

\subsection{Talos Description}

The Talos routine is a set of Fortran scripts developed to derive the SFH of an object based on its CMD and its MDF. It uses a set of isochrones chosen by the user, which at the moment includes the options Teramo (Pietrinferni et al. 2004), Dartmouth (Dotter et al. 2008), and Yonsei-Yale (Spada et al. 2013). With the isochrones chosen, it creates synthetic populations that are then contrasted with observations. To populate the synthetic models, Talos uses a Kroupa IMF (Kroupa 2001) and takes into account various properties of the input data to make the models directly comparable to the observations. These properties include distance, reddening, binary fraction, photometric errors, and completeness fractions. Then, the combination of synthetic populations that best matches the observed decontaminated CMD and MDF is selected as the SFH of the object. The comparison between synthetic and observed CMDs is done using Hess diagrams, in which each point represents the number of stars at a specific color-magnitude bin. Each time a combination of synthetic populations is created, the routine calculates the differences between the star counts in the model and the observations at 
each color-magnitude bin, along with the difference at each metallicity bin from the MDF. With these differences, Talos calculates a Poissonian chi-squared, whose minimization gives the resulting SFH (i.e., the model that best reproduces simultaneously the observed CMD and MDF).

\subsection{General Setup}

To run Talos and calculate the SFH, we have to determine a set of parameters associated with our data. These parameters are used to populate the synthetic models that are directly comparable to the data. From the entire CMD we used the photometry of a region that consisted of a magnitude range of 21-24.5 and a color range of $-0.5-1.15$, which we denominated the photometric CMD region (blue dashed box on Figure 1). The colors and magnitudes of the stars in the photometric CMD region are compared to the different models to fit the SFH. This region was chosen to avoid CMD locations significantly affected by completeness corrections or contamination sources, while maximizing our signal. Additionally, magnitudes brighter than $g=21$ were excluded to avoid regions of late stages of stellar evolution, where the different sets of isochrones we tried (Teramo, Dartmouth, and YonseiYale) differed significantly from each other. The individual bins that defined the Hess diagrams were chosen to have a size of 0.1 in magnitude and 0.025 in color. These dimensions were selected to be small enough to be able to trace the different features of the stellar populations in the CMD, and big enough to avoid significant shot noise in each bin. To construct the MDF of the data and the different models, Talos uses a region of the CMD where significant spectroscopic data exist, which we denominated the spectroscopic CMD region (green solid box on Figure 1). In our case, this region of the CMD had a $g-r$ color range of $0.5-1.25$ and a $g$-magnitude range of 18-20.5. Then, each time Talos generates a model stellar population, it constructs the associated artificial MDF with the metallicity values of all the stars from the model that lie in the spectroscopic CMD region. Analogously, the MDF of the data is constructed with the stars that have spectroscopic metallicity measurements and are also located within the spectroscopic CMD region.

The distance to Carina was determined using the magnitude of the RR-Lyrae and red clump stars from our data, and we obtained a distance modulus of $20.08 \pm 0.05$, consistent with recent values derived in the literature (Coppola et al. 2013; Weisz et al. 2014a; VandenBerg et al. 2015). The reddening was calculated on a star-by-star basis, using their equatorial coordinates and correlating them to the Schlegel maps (Schlegel et al. 1998). In this way, we correct for differential reddening throughout the galaxy.

To take advantage of the large spatial extent of our data, we determine the SFH independently for three different concentric regions of Carina:

Inner: $\quad 0<r / r_{\text {tidal }}<0.3$

Intermediate: $\quad 0.3<r / r_{\text {tidal }}<0.6$

Outer: $0.6<r / r_{\text {tidal }}<1.3$

The number of radial bins was chosen to include enough stars in each region to enable clear distinction of the different stellar populations in them. The corresponding dereddened CMDs of the stars from each of these three regions are shown on the first three panels of Figure 2, where we can see systematic differences between them. For each of these regions,
Talos was run using the corresponding Hess diagram (see left panels of Figure 7) and MDF (blue histograms of Figure 8).

To generate the synthetic stellar population models that are compared to the data, we used isochrones from the Dartmouth library (Dotter et al. 2008), which span a wide range of ages, metallicities, and alpha-element abundances. The range of metallicities used to define both the SFH and the MDF was $-3.5<[\mathrm{Fe} / \mathrm{H}]<-0.5$, which according to Koch's metallicity sample includes all the stars in Carina. The ages used ranged from $0.50 \mathrm{Gyr}$ to $14 \mathrm{Gyr}$, which is constrained by the age of the universe. To increase the resolution of the SFH, Talos interpolates between the different isochrones, and after this process we obtained a constant age spacing of $0.50 \mathrm{Gyr}$ and a constant metallicity spacing of 0.2 dex. Figure 6 shows a subsample of our set of isochrones plotted over the CMD of all the Carina stars we used in this study. This figure shows that the CMD region populated by the Carina data is completely contained by the region spanned by our isochrones. Therefore, the different stellar populations conforming to the SFH of Carina have ages and metallicities that are contained within the ranges defined above.

\subsection{Additional Input Files}

\subsubsection{Contamination}

The Carina $\mathrm{dSph}$ galaxy is located at relatively low galactic latitude $(b=-22.2 \mathrm{deg})$, and it spans a large area in the sky $\left(r_{\text {tidal }}=31\right.$ ! 0$)$. Thus, Carina's CMD is significantly contaminated by Milky Way foreground stars. To analyze the stellar populations in Carina, the foreground stars together with all the background sources have to be subtracted from the Hess diagrams. To account for their contribution, we used the sources located in the region beyond $2.0 \times r_{\text {tidal }}$ and extending to $3.0 \times r_{\text {tidal }}$, which we denominated the contamination region. The corresponding CMD of this region is shown on the rightmost panel of Figure 2. If we compare this CMD with the ones corresponding to the different Carina regions, we see that the contribution of Carina's stellar populations appears to be negligible in the contamination region. From the CMD of the contamination region, we constructed a contamination Hess diagram. In this way, the contamination Hess diagram does not include features from Carina's stellar populations, and it has enough stars to be a representative sample of the contamination sources.

To improve the reliability of our contamination Hess diagram, we applied a $6 \times 6$ kernel to smooth it. This significantly reduces the shot noise while still preserving the main features of the diagram. The smoothed contamination Hess was then scaled according to its spatial area and subtracted from the original Carina Hess diagrams. Normalized original and smoothed contamination diagrams are shown in Figure 3 and compared to the Carina Hess diagram.

\subsubsection{Artificial Star Test}

To calculate the photometric error and the completeness values as a function of color and magnitude, we ran artificial star tests on our photometry. We do this by adding stars to our individual images using the IRAF task ADDSTAR. Then, photometry was carried out following the same procedure used for the original images to measure the fraction of artificial stars recovered and the magnitude error. The error was calculated as the difference between the input magnitude and the one obtained with DAOPHOT. For each iteration, only 100 stars were added 

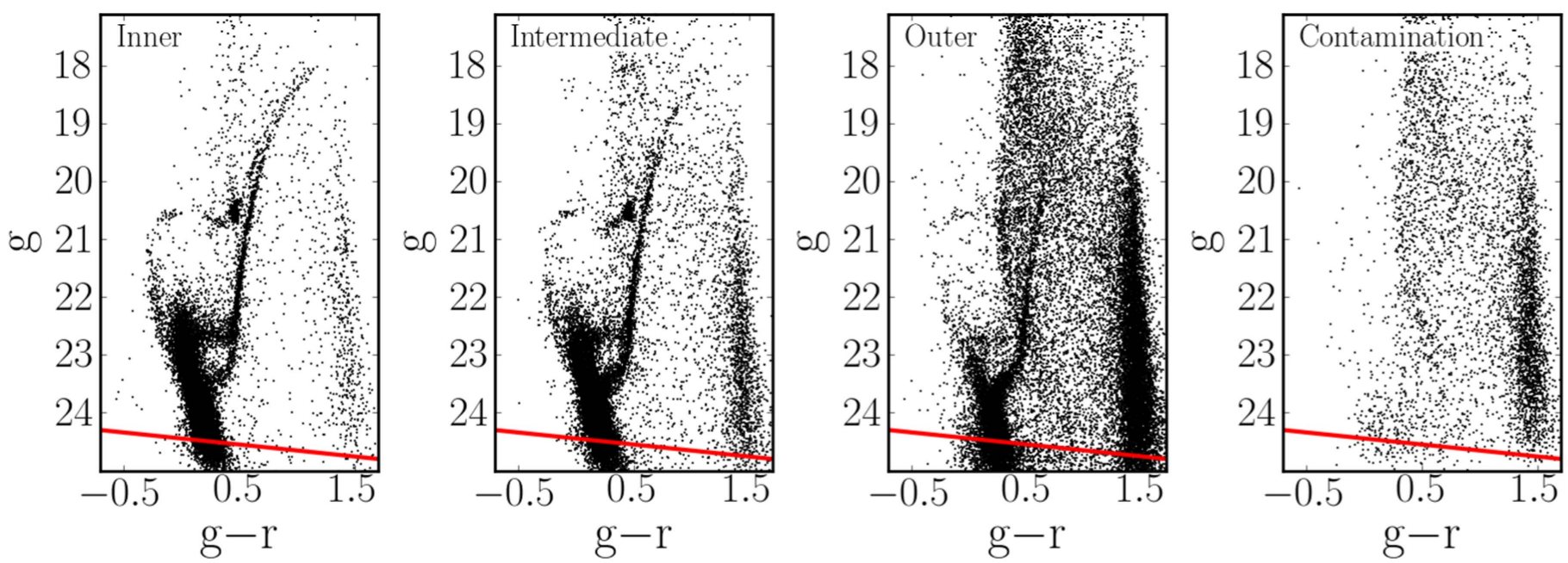

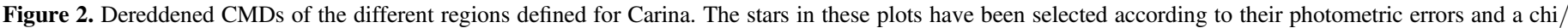

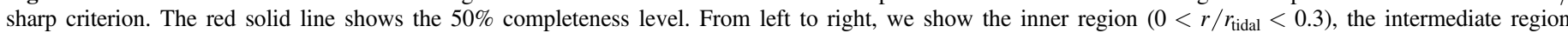

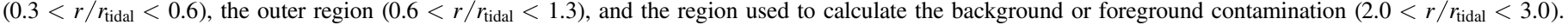
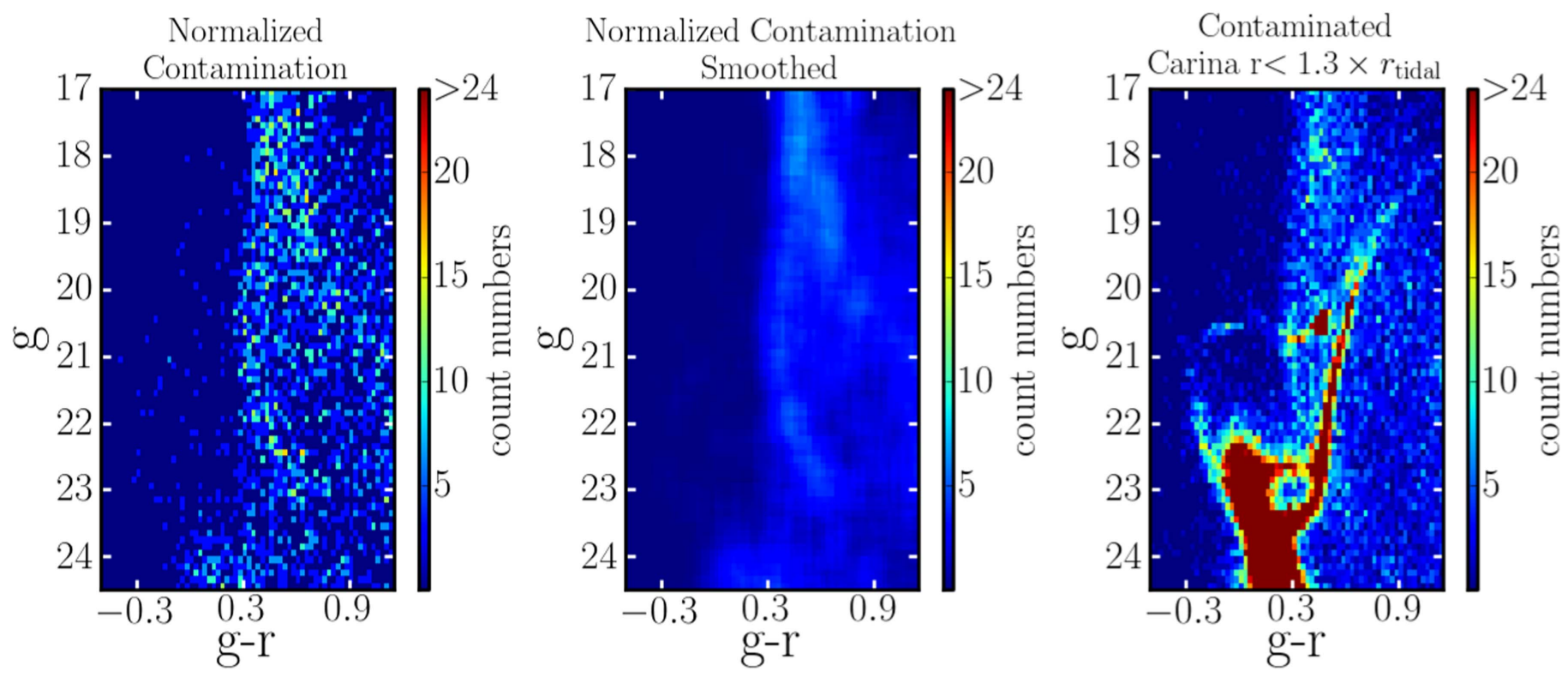

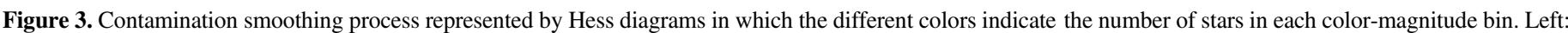

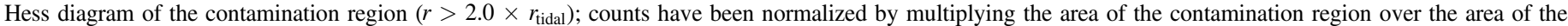

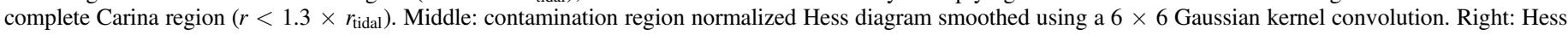

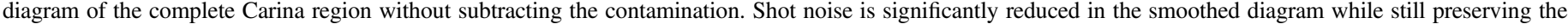
most important features that are also seen in the Hess diagram of Carina.

to avoid a significant alteration of the image crowding. A total of 280,000 stars were generated, covering all the regions of the CMD and the spatial extent of Carina inside $1.3 \times r_{\text {tidal }}$. The distribution of the input magnitudes generated had a maximum at $g \sim 25$. In this way, we increase the resolution of the completeness fraction determinations at the magnitudes where these fractions vary sharply. After performing the photometry on all the artificial stars, we obtained the completeness fraction as a function of magnitude and color for each region of Carina. These values were then used to correct the number counts in the Hess diagrams of Carina's different regions. Figure 4 shows the completeness fraction as a function of $g$ magnitude (left panel) and $r$ magnitude (right panel) for three different color ranges in the complete Carina region inside $1.3 \times r_{\text {tidal }}$.
The left-hand panel of this figure shows that we reach a 50\% completeness at $g \sim 24.5$, with values at redder colors being slightly higher. This implies that we have completeness fractions higher than $50 \%$ for the entire photometric CMD region defined in Section 3.2. With the artificial star test, we also estimated the magnitude error of our photometry, calculated using the absolute value of the difference between input and recovered magnitudes of the artificial stars. The median of this value was calculated for each magnitude bin, and these numbers were then used to reproduce the photometric errors on the synthetic stellar populations. Figure 5 shows the median and one-sigma intervals of the magnitude error estimation as a function of $g$ (left) and $r$ (right) magnitudes. 


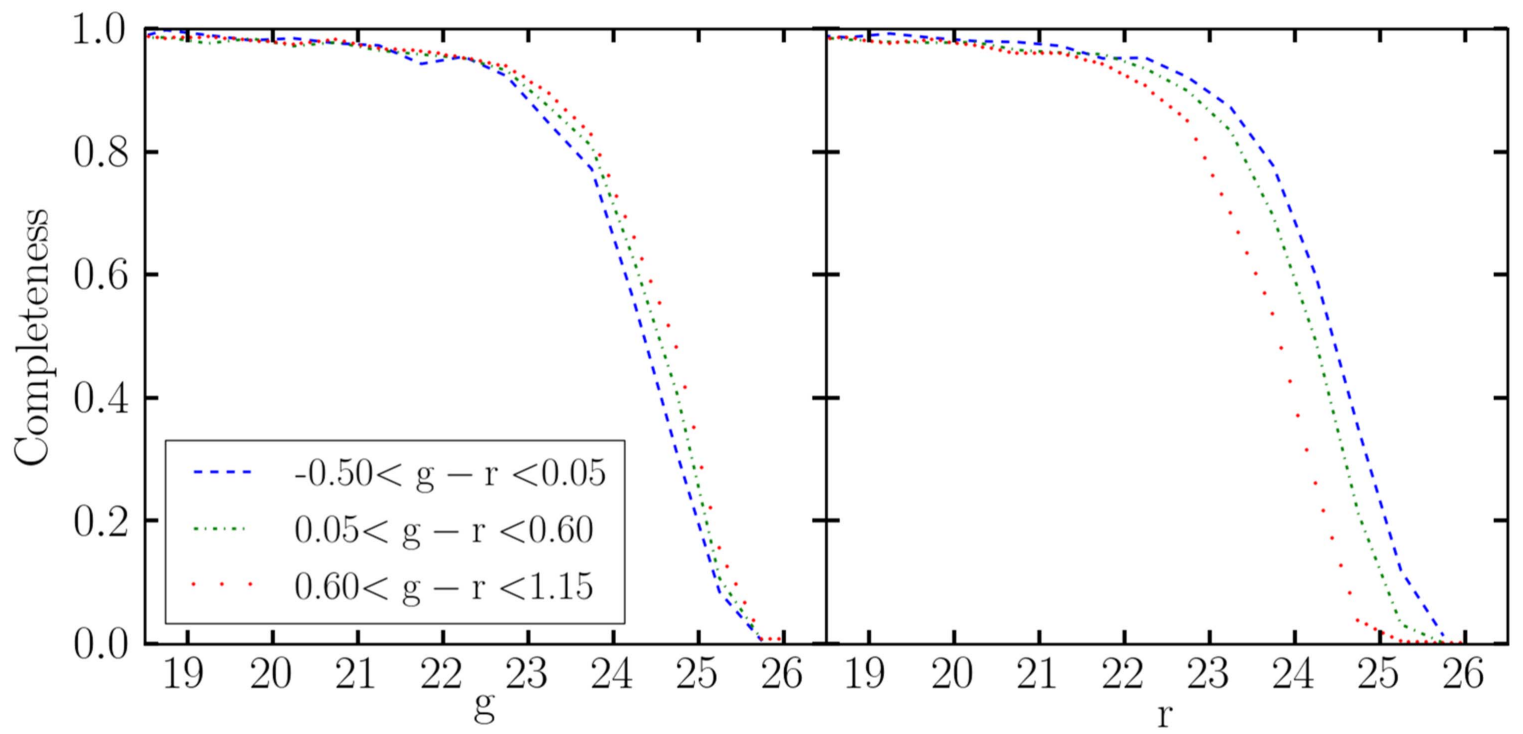

Figure 4. Completeness vs. magnitude. Fraction of stars recovered by the artificial photometry as a function of input $g$ (left) and $r$ (right) magnitudes. The different lines show stars at different input colors.

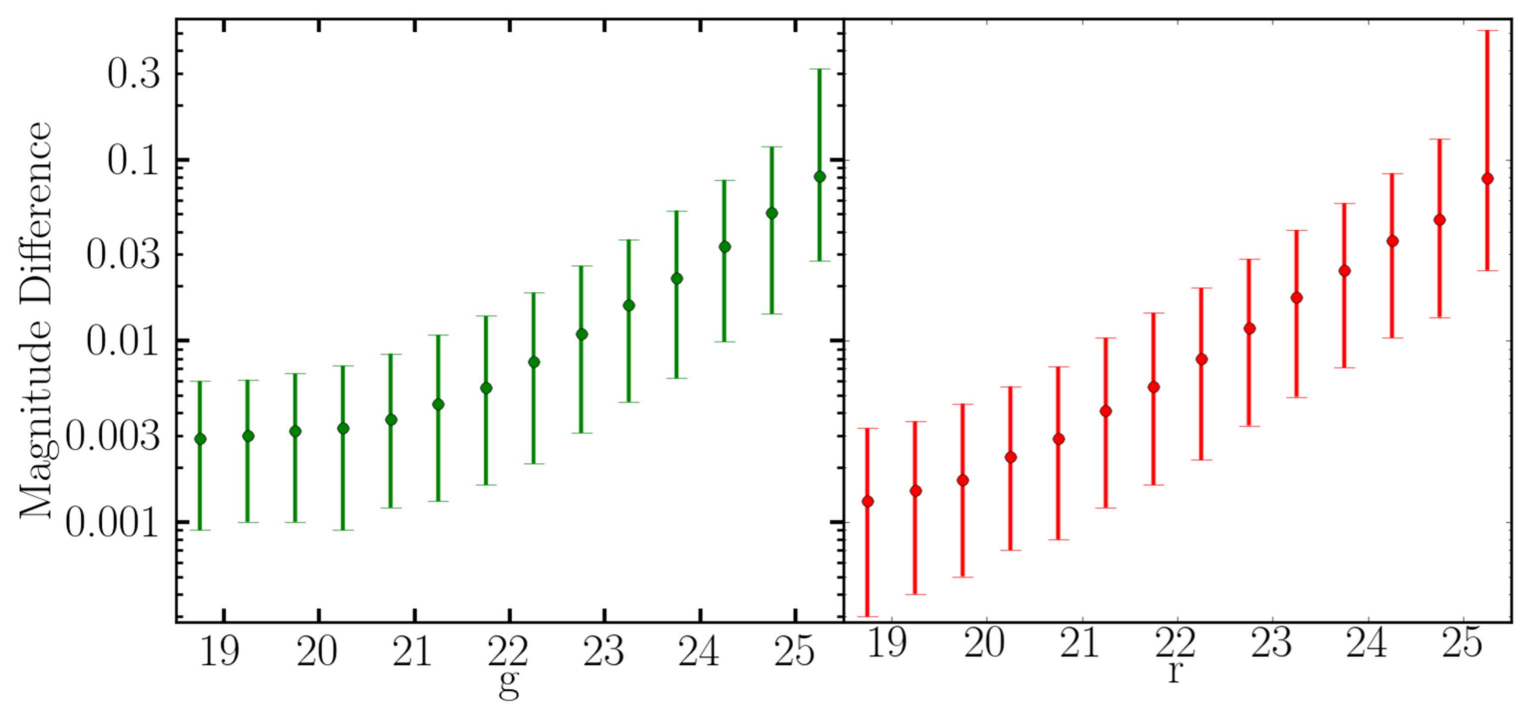

Figure 5. Photometric error vs. magnitude. The errors are estimated as the absolute values of the difference between input and recovered magnitude for the artificial star test as a function of input $g$ (left) and $r$ (right) magnitudes. For each magnitude bin, we plot the median of the absolute value of the difference along with the onesigma interval.

\section{RESULTS}

The SFH for each of the Carina regions defined in Section 3.2 was determined by comparing our data with a set of synthetic stellar populations, which were created using a set of Dartmouth isochrones. To generate the models that are compared to the data, Talos uses the binary fraction of the system as an input. Since this number has not been determined observationally for Carina, we allow the code to find the best choice. For this, we performed various runs of Talos, using different binary fractions each time. After this, we chose a binary fraction value ${ }^{8}$ for Carina of 0.4 , because this value

\footnotetext{
8 Even though the binary fraction is, in principle, a function of the stellar mass (e.g., Kouwenhoven et al. 2009), the vast majority of the stars we are using to fit the SFH in Carina are close to the main-sequence turnoff, and therefore their masses are closely clumped in the range $0.7-0.9 M_{\odot}$.
}

produced the Hess diagram models that most resembled the data. This binary fraction is consistent with values obtained in other dwarf galaxies (e.g., McConnachie \& Côté 2010; Geha et al. 2013).

Figure 7 shows the Hess diagram of the data (left panels) and the best-fit model (right panels), as well as the residuals for each Carina region. We see in this figure that, for all regions, the best fit is an accurate representation of the observations, with residuals lower than one sigma for $80 \%$ of the colormagnitude bins and lower than $2 \sigma$ for $\sim 99 \%$ of the bins. The largest systematic differences from the data and best models lie in the main-sequence color for the inner region, which is bluer for the model than for the data, and the main-sequence width for the outer region, which is larger for the model than for the data. The reason for these systematics may be attributed to a combination of an offset between the assumed binary 


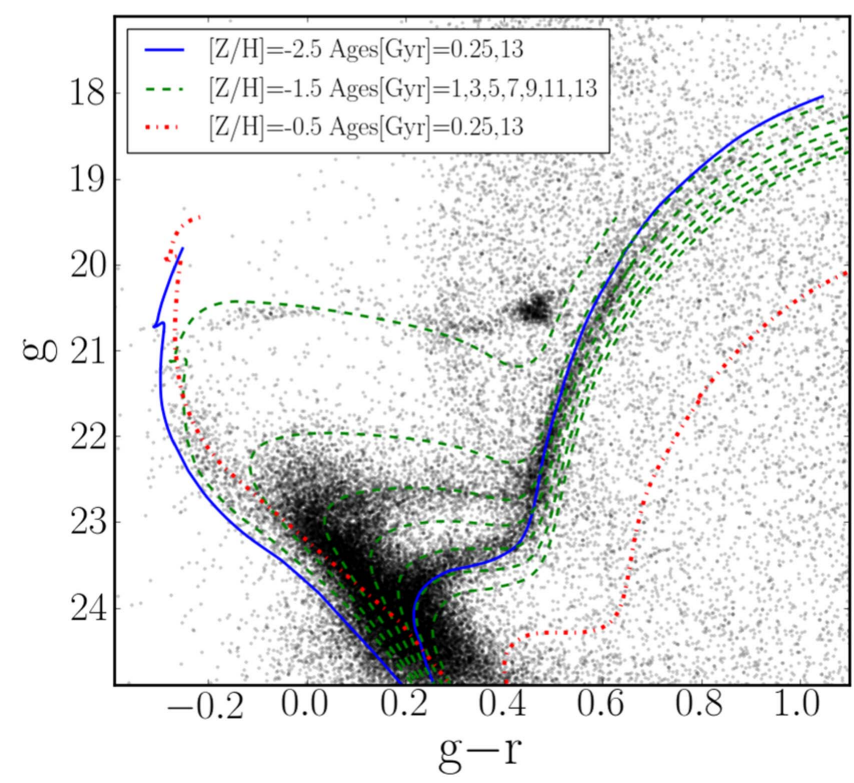

Figure 6. CMD of all the stars in Carina used in this study along with a subsample of the Dartmouth isochrones used to derive the SFH. We show isochrones at three different metallicities: $-2.5,-1.5$, and -0.5 . For the isochrones with $[\mathrm{Z} / \mathrm{H}]=-1.5$, we show isochrones for various ages from 1 to 13 Gyr. For the other two isochrones, we just show the lower and upper limits of the ages used in this study, which are 0.25 and $14 \mathrm{Gyr}$, respectively. This plot shows that the CMD region of our Carina data is completely contained within the region spanned by our set of isochrones.

fraction distribution and the real ones, an incorrect estimation of the magnitude error as a function of magnitude, uncertainties in the calibration of the data, and an error in the isochrone models used. Even though the specific origin of our systematics could not be identified, we do not deem them significant since they are in almost all cases similar to the expected random errors.

The model Hess diagrams reveal the presence of an old and an intermediate-age population for all Carina regions, but the relative importance of the intermediate-age population decreases as we move to larger radii. A combination of these two populations correctly reproduces the main features of Carina's CMDs, such as main-sequence turnoff positions, the gap separating the two main subgiant branches, and the width of the RGB. There are also hints of a young population in all of Carina's regions, accounting for $1 \%-2 \%$ of the stellar mass, and their authenticity will be analyzed in Section 5 .

Figure 8 shows the comparison between data and best-fit model for the MDFs. The histograms in this figure include only stars from the spectroscopic CMD region (defined in Section 3.2). It is important to note that the model histograms in this figure (blue histograms) are different from those in Figure 11, because the MDFs presented in those plots include the stars in the model from the entire CMD region.

MDFs of the models closely reproduce the observations for all Carina regions, with differences between the two always within the uncertainties. This figure indicates that the average metallicity of the RGB stars in Carina decreases with radius, showing a large spread at all regions.

The full SFH derived for each Carina region is shown in Figure 9. This plot displays the stellar mass formed at each age-metallicity combination and clearly shows the presence of two main episodes of star formation separated by a star- formation temporal gap. The old episode occurred more than $10 \mathrm{Gyr}$ ago, and its stars have very low metallicity $(-3.0<[\mathrm{Fe} / \mathrm{H}]<-2.0)$. The intermediate-age episode started $\sim 8$ Gyr ago and stopped $\sim 2$ Gyr ago, increasing its metallicity from $\sim-2.0$ to $\sim-1.0$ in that period. This shows that the average stellar metallicity at the moment the intermediate-age episode began is consistent with the stellar metallicity of the last stars formed in the old episode, and therefore there is a continuous transition in stellar metallicity between the two episodes. The temporal gap of star formation separating the two main bursts represents an epoch where no significant star formation took place. This gap lasted for a few billion years between $\sim 10$ and 8 Gyr ago.

The central age and metallicity of the two main episodes are consistent through all Carina regions, but their relative importance varies from the inner to outer regions. The inner region is dominated by the intermediate-age episode while still having an important contribution from the old one. The middle region displays both episodes with similar relative importance. Finally, the outer region is composed mainly of old stars with a very small contribution from the intermediate-age episode.

There is no clear evidence for a young (age $<2$ Gyr) episode in Figure 9, which could indicate that there are no young stars in Carina, or that their contribution is negligible compared to the two main episodes. The presence of a young population has been claimed by previous studies (e.g., Hurley-Keller et al. 1998; Monelli et al. 2003), so in the following section we will analyze the authenticity of the young population in Carina and the distinction between these stars and blue stragglers.

As shown by Aparicio \& Hidalgo (2009), the main source of statistical uncertainties in $\mathrm{SFH}$ results derived from the synthetic CMD method is the specific choice of the grids that define the Hess diagrams, MDFs, and the resulting SFHs. The general hypothesis is that there is a loss of information produced by discretizing the values contained in these distributions, according to the arbitrary grids defined by the user. To account for this source of error, for each of the regions we defined for Carina (inner, intermediate, and outer), we carried out 32 runs of Talos in it. Each time a trial of Talos was performed, we shifted the grid defining the input photometry or spectroscopy grid or the grid defining the resulting SFH. Then the final SFH of a given Carina region was determined as the average of the solutions obtained in all the trials, while the associated errors were calculated as the standard deviations of the different solutions.

The SFH as a function of age is shown in Figure 10, where we can see the same general features as in Figure 9. There are two main episodes separated by a temporal gap in star formation, and the relative importance of the old episode increases with radius, indicating a positive age radial gradient present in Carina, which is steeper between the middle and outer regions. Figure 11 shows the SFH as a function of metallicity (or chemical enrichment history). In these plots we can see that the metallicity distribution is similar in the inner and intermediate regions, but the average metallicity in the outer region is systematically lower than in the rest of the galaxy, indicating a negative metallicity gradient. From the results presented in this figure, we calculated that Carina has a mean metallicity of $[\mathrm{Fe} / \mathrm{H}]=-1.81 \pm 0.29$ and a spread of $\sigma=0.54$ dex. The SFH of Carina that we derived is able to reproduce both the large dispersion MDF and the narrow RGB seen in the Hess diagrams. This shows that these two features in 

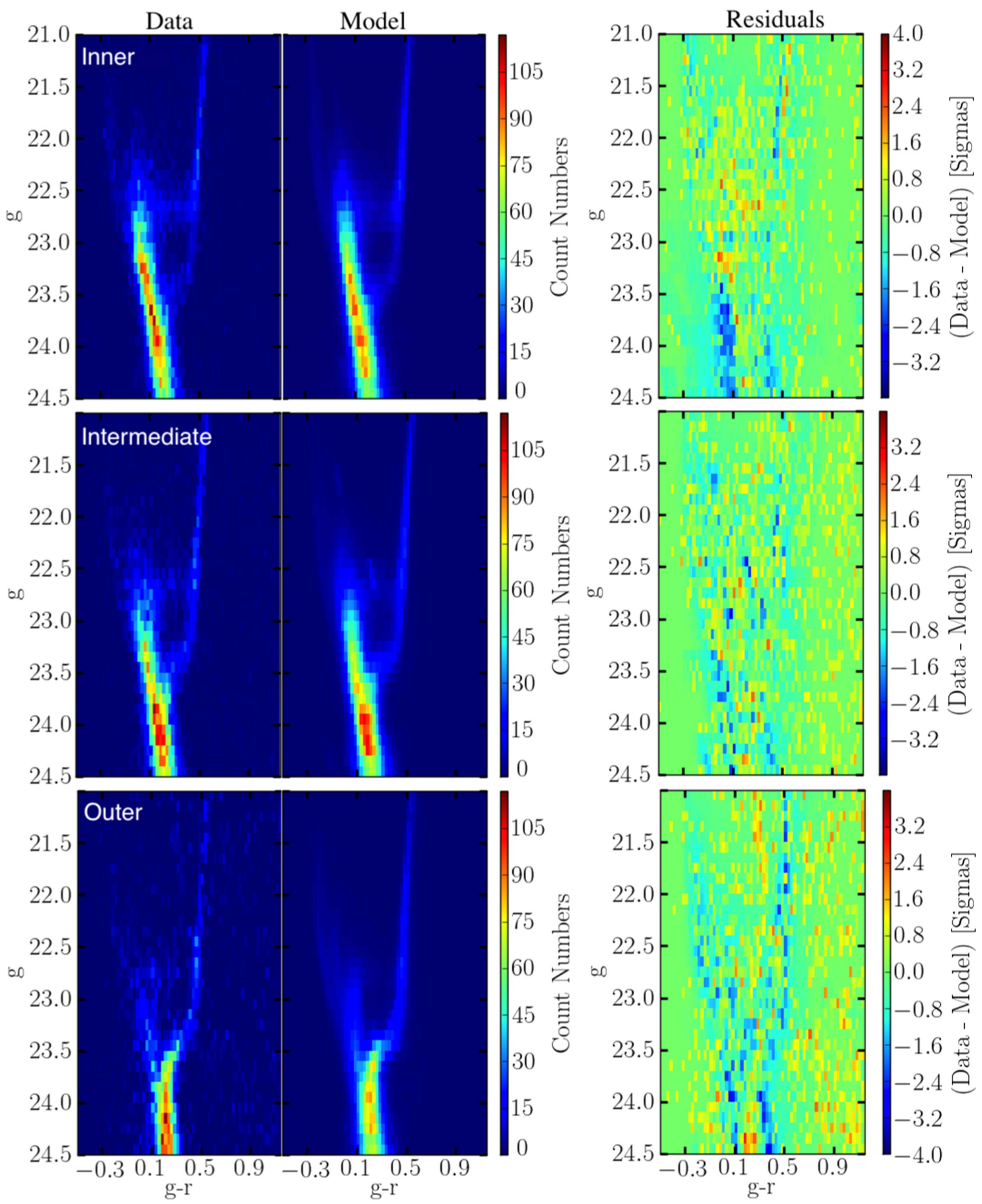

Figure 7. Comparison of observed and model CMDs represented by Hess diagrams. Colors in plots represent number of stars in each CMD region. Each panel shows at left the CMD of the data, in the middle the best-fit model CMD, and at the right the residuals calculated as (model-observed)/errors. Top: inner region corresponding to $0<r / r_{\text {tidal }}<0.3$. Middle: intermediate region corresponding to $0.3<r / r_{\text {tidal }}<0.6$. Bottom: outer region corresponding to $0.6<r / r_{\text {tidal }}<1.3$.

Carina are consistent, and their implications will be interpreted in the following section. From the SFH derived for Carina, we also estimated the total stellar mass formed in the galaxy, obtaining $1.60 \pm 0.09 \times 10^{6} M_{\odot}$ within $1.3 \times r_{\text {tidal }}$ and $1.45 \pm 0.12 \times$ $10^{6} M_{\odot}$ within the nominal $r_{\text {tidal }}$. The latter value is larger than previous values derived for Carina (e.g., de Boer et al. 2014 derived a value of $1.07 \pm 0.08 \times 10^{6} M_{\odot}$ within the nominal $\left.r_{\text {tidal }}\right)$. With these results we also estimated the total visual luminosity of Carina as $L_{\mathrm{V}}=7.3 \pm 0.4 \times 10^{5} L_{\odot, \mathrm{V}}$.

\section{DISCUSSION}

\subsection{Young Population versus Blue Straggler Population}

The star-formation history of Carina clearly shows at least two different episodes. Even though a young population is not clearly evident, as seen in Figures 7, 9, and 10, a small but not negligible contribution of young stars (age $<2$ Gyr) might also be present in Carina, which can account for $1 \%-2 \%$ of the total stellar mass according to our SFH derivation. Different studies 

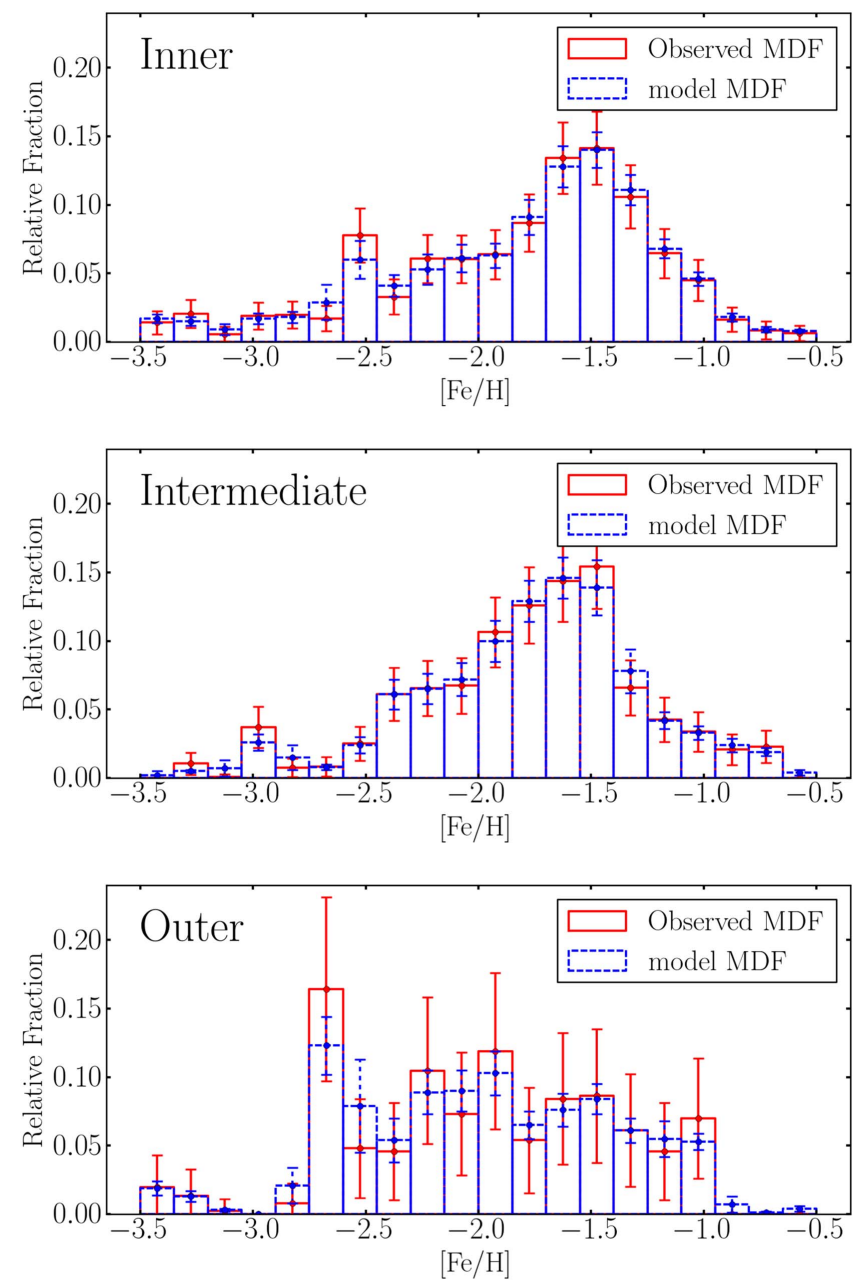

Figure 8. MDF of observations and model in different regions of Carina. These distributions include only the stars from the spectroscopic CMD region (defined in Section 3.2). Top: inner region corresponding to $0<r / r_{\text {tidal }}<0.3$. Middle: intermediate region corresponding to $0.3<r / r_{\text {tidal }}<0.6$. Bottom: outer region corresponding to $0.6<r / r_{\text {tidal }}<1.3$.

have claimed the presence of a young population in Carina (e.g., Hurley-Keller et al. 1998; Mateo et al. 1998; Monelli et al. 2003). However, there exists the possibility that these stars correspond to blue stragglers mistakenly classified as young stars. Blue straggler stars are coeval to a given stellar population, but bluer and brighter than its main-sequence turnoff. Thus, these stars can populate the same region as young stars in a CMD, and therefore their differentiation is not straightforward.

To analyze the authenticity of the young population of Carina, we developed a method to discriminate blue straggler counts from young star counts. To apply this method, we start by calculating the number of stars in Carina located in a region brighter and bluer than the main-sequence turnoff of the intermediate-age population. In principle, we do not know if these stars are blue stragglers or young stars, so we will denominate the sum of both contributions as blue plume counts. The goal is to compare the blue plume fraction of Carina with the blue straggler fraction found in a subsample of the local dSph galaxies used in Santana et al. (2013). For those galaxies, the authors showed that the stars brighter and bluer than the main-sequence turnoff are genuine blue stragglers. Additionally it was shown that local dSphs share a common
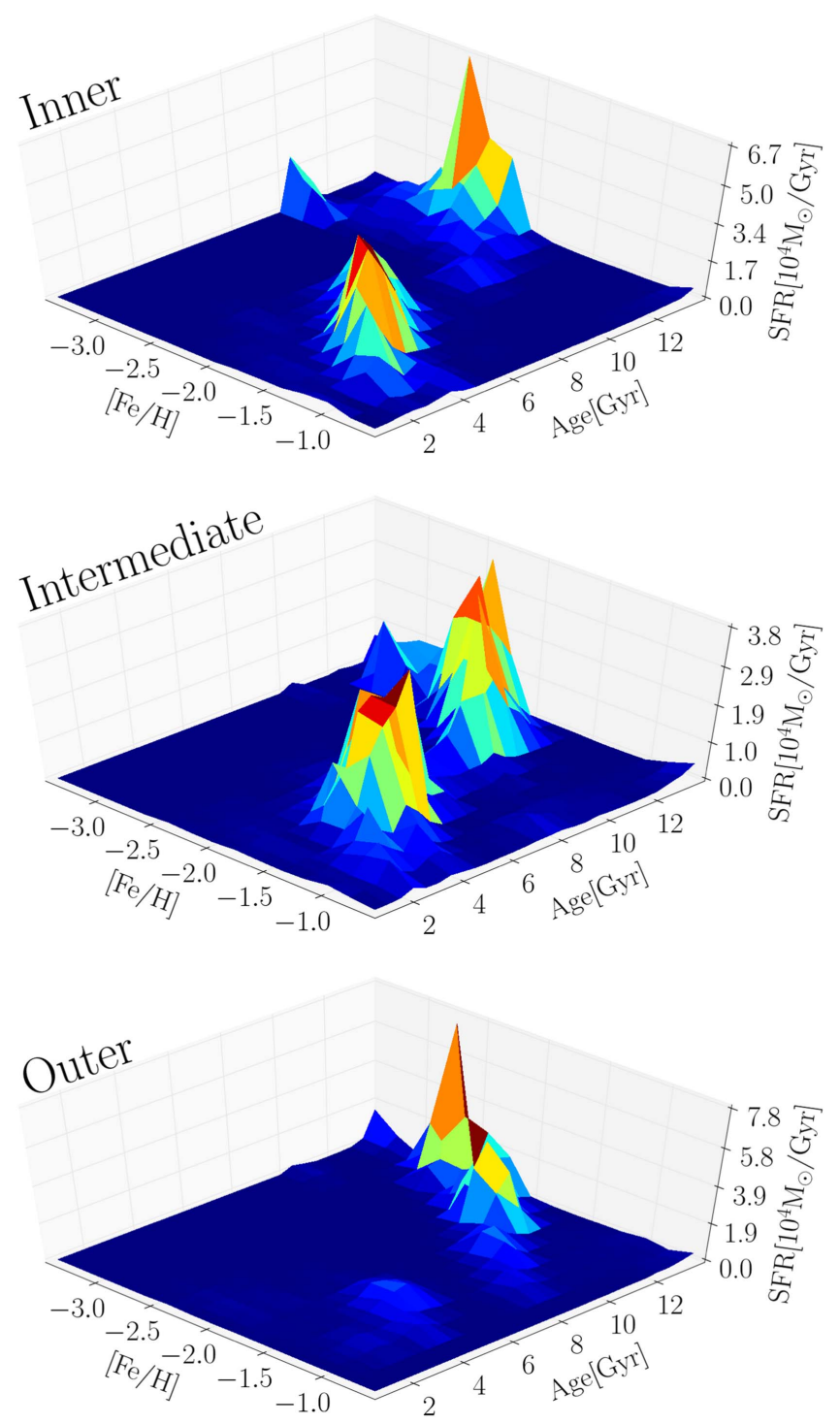

Figure 9. Three-dimensional SFH of different regions of Carina. The color and height of each bin show the stellar mass formed at that age and metallicity. Top: inner region corresponding to $0<r / r_{\text {tidal }}<0.3$. Middle: intermediate region corresponding to $0.3<r / r_{\text {tidal }}<0.6$. Bottom: outer region corresponding to $0.6<r / r_{\text {tidal }}<1.3$.

fraction of blue straggler stars (Momany et al. 2007; Santana et al. 2013). Therefore, by comparing the blue plume fraction of Carina with the blue straggler fraction in these local dwarf galaxies, we aim to check what contribution of the blue plume fraction of Carina comes from blue stragglers and what fraction from young stars. Using this result, if the fraction of stars in the blue plume of Carina is similar to the blue straggler fractions in the other local dSphs, then they are most likely dominated by blue stragglers. But if this fraction is considerably higher than the average blue straggler fractions in other dSphs, then the blue plume is probably dominated by young stars. Details on how the blue plume and blue straggler fractions were calculated are given in the Appendix.

The final blue straggler fraction for each of the comparison local dSphs is shown in Figure 12, along with the blue plume fraction found for Carina. As we can see in this plot, local dSphs share a common blue straggler fraction (as found in Santana et al. 2013). It can also be seen in Figure 12 that the 

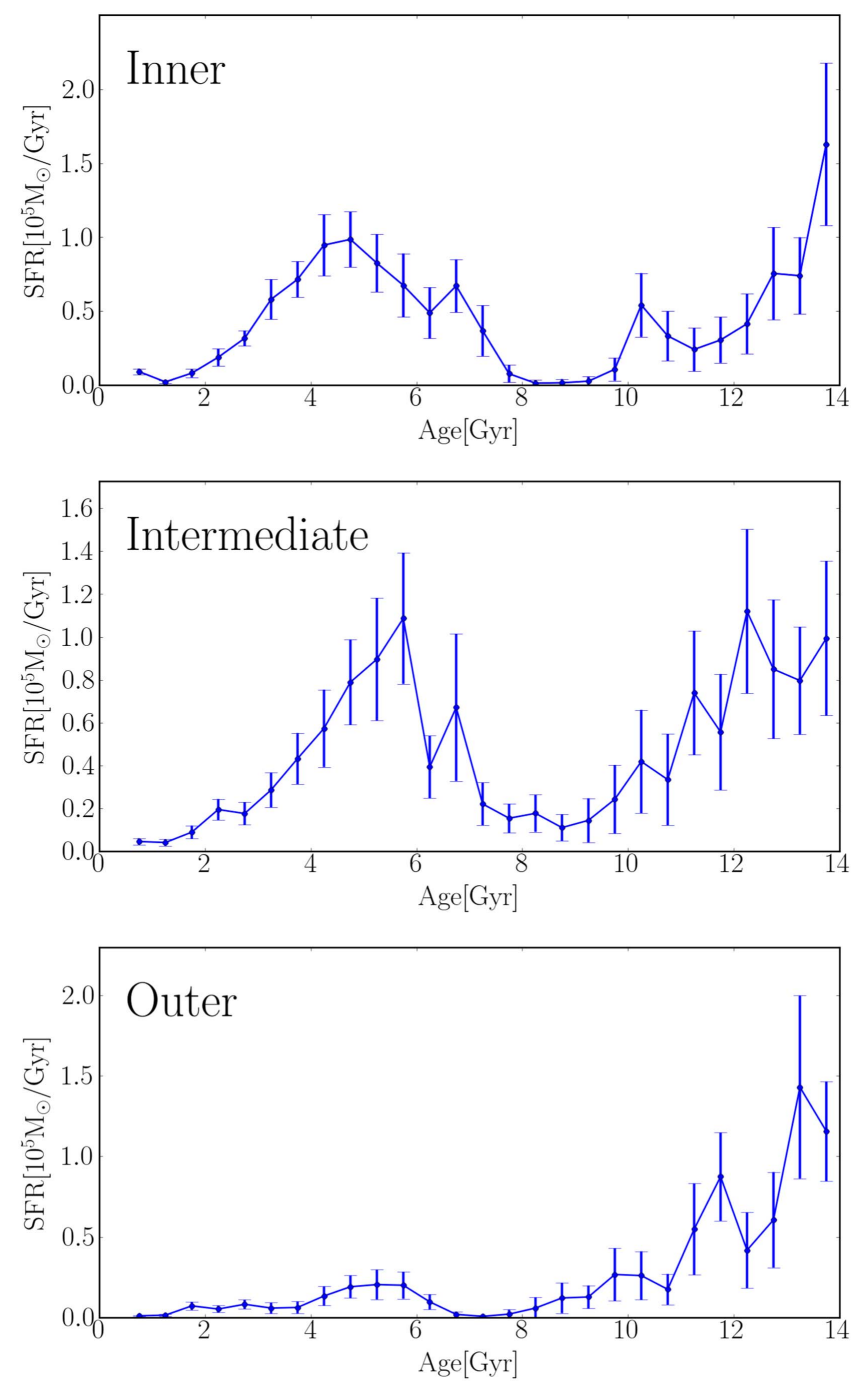

Figure 10. Star-formation rate as a function of age for different regions of Carina. Top: inner region corresponding to $0<r / r_{\text {tidal }}<0.3$. Middle: intermediate region corresponding to $0.3<r / r_{\text {tidal }}<0.6$. Bottom: outer region corresponding to $0.6<r / r_{\text {tidal }}<1.3$.

difference between the blue plume fraction of Carina and the blue straggler fraction in our dwarf galaxies is less than the standard deviation of the blue straggler fraction. This shows that the number of stars in the blue plume of Carina's CMD is completely consistent with the number of blue stragglers found in other local dSphs; hence, we claim that there is no considerable contribution of young $(<2 \mathrm{Gyr})$ stars in Carina. We thus conclude that the Carina dSph galaxy had only two episodes of star formation and the small contribution of young stars derived by Talos are due to misclassified blue straggler stars.

It is worth noting that genuine young stars have been claimed in previous studies about the SFH of Carina (e.g., Hurley-Keller et al. 1998; Monelli et al. 2003). However, the relative fraction that these stars represent from the total stellar mass of the system was not quantified in these studies. Furthermore, the arguments used by these authors to claim that these stars are young and not blue stragglers are based on the hypothesis that these stars are much more numerous than the
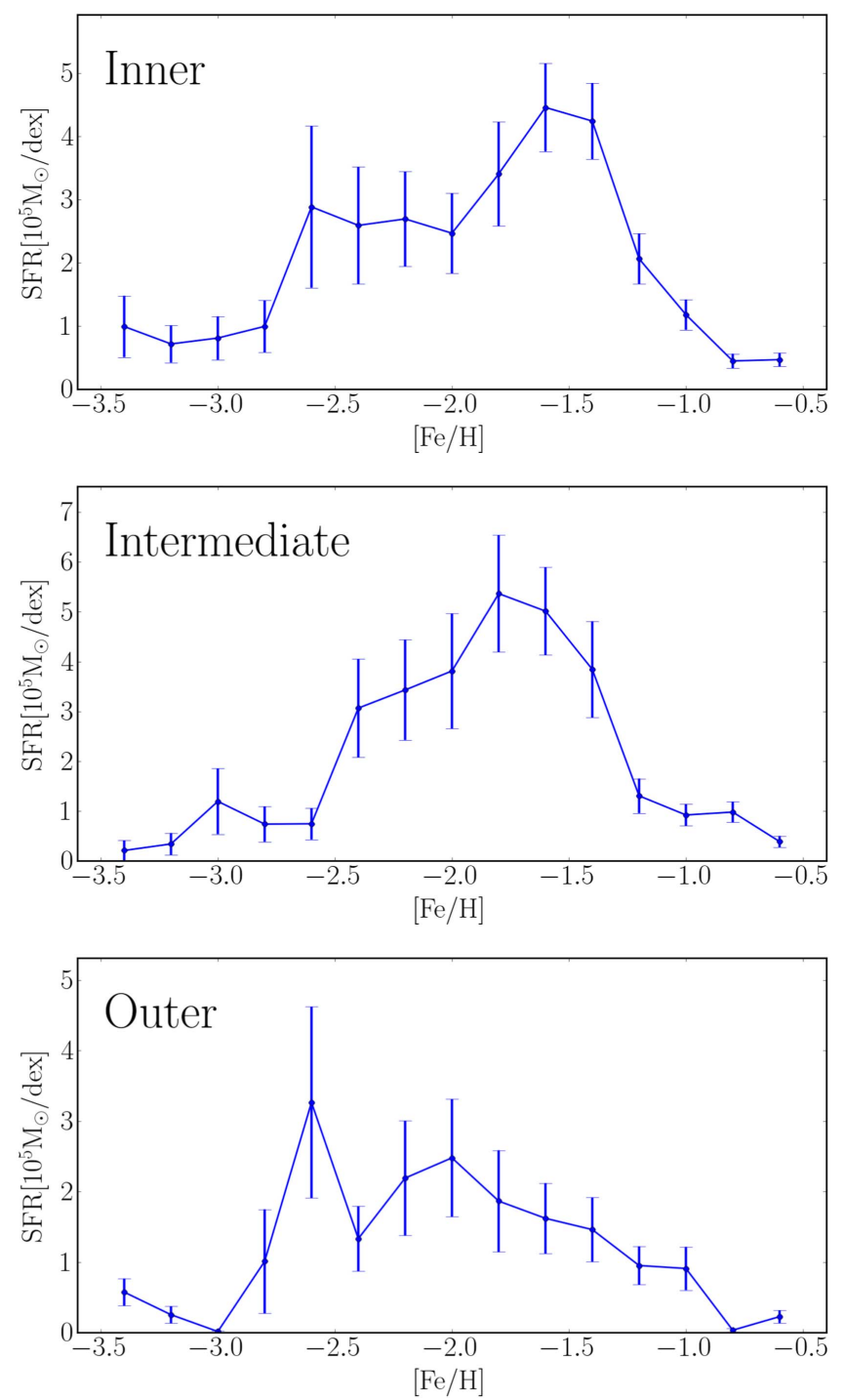

Figure 11. Star-formation rate as a function of metallicity for different regions of Carina. Top: inner region corresponding to $0<r / r_{\text {tidal }}<0.3$. Middle: intermediate region corresponding to $0.3<r / r_{\text {tidal }}<0.6$. Bottom: outer region corresponding to $0.6<r / r_{\text {tidal }}<1.3$.

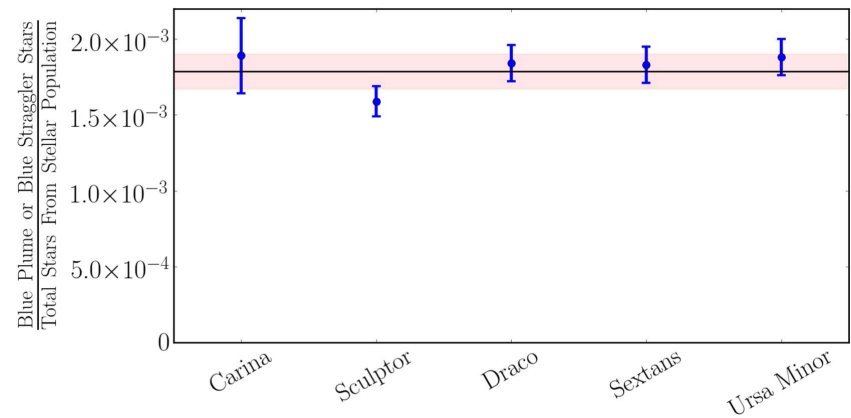

Figure 12. Blue straggler or blue plume fractions for the different galaxies. For Carina, we show the number of blue plume stars over the number of RGB stars from the intermediate-age population. These blue plume counts include, in principle, young stars and blue stragglers formed from the intermediate-age stellar population. For all the other galaxies, we show the blue straggler fraction calculated as the number of blue stragglers over the number of RGB stars. The black solid line indicates the average blue straggler fraction for all the galaxies, and the red region shows the area located at a maximum of one standard deviation difference from the average. 
expected number of blue stragglers in globular clusters. There are two possible problems in associating the properties of blue stragglers in globular clusters to the blue stragglers in the Carina dSph galaxy. First, Santana et al. (2013) showed observationally that the fraction of blue stragglers in dwarf galaxies can be of the order of $10 \times$ higher than those in globular clusters, which, according to the authors, is because of the higher prevalence of binaries from which blue stragglers are formed in the low-density environments present in local dSph galaxies. Second, Milky Way globular clusters are old stellar populations (older than $\sim 10-11$ Gyr according to recent reviews such as Gratton et al. 2012), whereas Carina contains an old and an intermediate-age episode. Main-sequence stars from the intermediate-age population have a range in luminosity and mass that extends to higher values than the one of old stars. Therefore, the merger or mass transfer between the intermediate-age stars from Carina should produce blue stragglers brighter than the ones formed in globular clusters. Thus, we claim that the number of blue stragglers in Carina brighter and bluer than the intermediate-age main-sequence turnoff is not expected to match the properties of blue stragglers in globular clusters.

Further complementary observations can significantly help to confirm the nature of the stars in the blue plume of galaxies like Carina. For example, Gosnell et al. (2014) have confirmed the presence of three hot and young white dwarfs as companion stars in binary systems for three blue straggler stars in the open cluster NGC 188. These white dwarfs would correspond to former RGB or AGB stars that transferred mass in the recent past to their companion stars in the binary system, hence transforming them into blue stragglers. These stars were detected as a high-significance ultraviolet excess using three narrow-band filters from the Advance Camera Survey on board $H S T$. The spectral energy distribution derived from the photometry of these stars was well fit by the sum of the contributions of a single blue straggler and a white dwarf of different temperatures for the three cases. That study represents the first direct determination of the formation mechanism for individual blue stragglers, and further observations of this type can significantly help to confirm or rule out the blue straggler nature of different blue plume populations.

\subsection{SFH of Carina: Internal Evolution versus External Influence}

Carina is a local dSph galaxy whose SFH has raised great interest since its discovery (Cannon et al. 1977), mainly because it is the only dSph in the Local Group showing clear multiple main-sequence turnoffs. Additionally, Carina displays signs of tidal influence from the Milky Way (Muñoz et al. 2006; Battaglia et al. 2012). In this context, one of the key questions regarding Carina's SFH is how much it is governed by interactions with the Milky Way, as opposed to being the result of internal evolution.

The depth and quality of the data presented in this work represent a key opportunity for unraveling the nature of Carina's SFH. We have $g$ and $r$ Sloan band photometry reaching a $50 \%$ completeness at $g \sim 24.5$, more than one magnitude fainter than the oldest turnoff. In addition, the field of view of our observations encompasses an area of $\sim 2 \mathrm{deg}^{2}$, which translates into full coverage out to $1.3 \times r_{\text {tidal }}$ and partial coverage to $3.0 \times r_{\text {tidal }}$. The combination of depth and coverage of the data is important for tracing the oldest stellar population, which extends even farther than $r_{\text {tidal }}$ and has a faint main-sequence turnoff.

In this work, we have used the full Hess diagrams of different regions in Carina along with the MDF from public spectroscopy (Koch et al. 2006), and we compared them to synthetic stellar populations using the routine Talos (de Boer et al. 2012). With this method, we obtained an SFH composed of two episodes separated by a temporal gap in star formation. The episodic behavior of the SFH of Carina is consistent with what had been claimed by various previous studies (e.g., Smecker-Hane et al. 1996; Hurley-Keller et al. 1998; de Boer et al. 2014). The first episode corresponds to an old (age $\sim 10-13.5 \mathrm{Gyr}$ ) population with metallicities in the range of $[\mathrm{Fe} / \mathrm{H}] \sim-3.0$ to -2.0 , and the second one is an intermediate-age (2-8 Gyr) episode with metallicity increasing with age from $[\mathrm{Fe} / \mathrm{H}] \sim-2.0$ to $[\mathrm{Fe} / \mathrm{H}] \sim-1.0$. The old and intermediate-age episodes correspond to $54 \pm 4 \%$ and $45 \pm 4 \%$ of the stellar mass, respectively. This is the first study of the SFH of Carina where the old episode is found to form the majority of the population. This result is in agreement with what is often found for other local dSphs (e.g., Grebel 1999; Tolstoy et al. 2009). The different relative fractions obtained for the old population of Carina might be a result of our deeper data. The proper photometric characterization of the old main-sequence turnoff is given by the fact that it is more than one magnitude brighter than the 50\% completeness level of our photometry (see Figure 1). This has a stronger effect in the outermost regions of Carina where the old population is relatively more important: we obtained higher relative fractions for the old population than previous studies simply because we can detect them more reliably and to larger radii. Considering that Carina might have experienced signifiant mass loss in the past due to tidal influence (e.g., Muñoz et al. 2006) and that this influence is stronger for more extended stellar components (Sales et al. 2010), then the older stars would have preferentially been removed from Carina. Given this, the fraction of old stars presented here would represent the current value and a lower bound to the fraction of old stars formed in the galaxy. If this is the case, then the difference between the number of stars from the old and intermediate-age populations would be even larger, and the prevalence of the old stellar population would be clearer. Separating these two main episodes is an epoch consistent with no star formation at all, occurring between 8 and $10 \mathrm{Gyr}$ ago, as previously observed by various studies (e.g., Hurley-Keller et al. 1998; Bono et al. 2010; de Boer et al. 2014). Finally, the data show evidence that could be interpreted as a small contribution of young stars (less than $2 \%$ of stellar mass). However, as we concluded in Section 5.1, we believe that these stars are most likely dominated by blue stragglers.

The SFH derived for Carina in this work produces good matches with both the input CMDs and MDFs, as shown by Figures 7 and 8, respectively. This implies, for example, that a metallicity distribution with a large dispersion and a narrow color distribution can be completely consistent with a complex SFH, with two important episodes separated by a couple of gigayears, which indicates a large metallicity-age degeneracy.

If we analyze the SFH of the inner, intermediate, and outer regions of Carina separately, we see that all the regions show the same central ages and metallicities for the two episodes. However, as we go to larger radii, the relative importance of the old episode increases. This produces a positive age radial 
gradient and a negative metallicity radial gradient, which are steeper between the intermediate and outer regions. The gradient in the SFH of Carina is consistent with what had already been reported by various previous studies (e.g., Battaglia et al. 2012; de Boer et al. 2014; McMonigal et al. 2014).

One consequence of the spatial variation of Carina's SFH is the fact that the intermediate-age population is practically negligible at large radii. The lack of intermediate-age stars in the outer region of Carina suggests that, once this population started forming, tidal forces from the Milky Way, or any other physical process ${ }^{9}$, were not strong enough to move a significant fraction of these stars to the outer region. Otherwise, a large number of intermediate-age stars would be present in the outskirts of Carina, and thus we would not observe such a strong break in the SFH and MDF of Carina between the intermediate and outer regions. Since the radial trends in Carina do not seem to be governed by the tidal influence of the Milky Way, we suggest that this hints that the SFH is not dominated by tidal forces either. In this line, the fact that we find two episodes of star formation in Carina instead of three, as has often been claimed (Hurley-Keller et al. 1998; Rizzi et al. 2003; Pilkington \& Gibson 2012; McMonigal et al. 2014), makes it harder to explain the star-formation episodes in Carina as being the result of periodic close passages to the Milky Way (as done in Pasetto et al. 2011), even more so since proper motions suggest that the orbital period of Carina is close to 2 Gyr (Piatek et al. 2003; Pasetto et al. 2011).

Another mechanism proposed to explain the SFH of Carina is gas accretion from the Milky Way (e.g., Lemasle et al. 2012; de Boer et al. 2014). In this view, the first episode would have ended because of gas depletion, and subsequent star formation would have been stopped for a few gigayears until the dwarf galaxy renewed its gas content thanks to an inflow from the Milky Way. Even though this is a plausible scenario, the infalling gas should have had the same metallicity as the stars from the end of the first episode to reproduce the agemetallicity relation in Carina. A simpler way to explain why the metallicity of the stars at the end of the first episode coincides with that of the stars at the beginning of the second episode is to assume that the gas that formed the second episode in Carina was enriched within the same galaxy by the first generation of stars.

Internal evolution would also be consistent with the mean metallicities and ages at different Carina regions, which according to what we see in Figure 9 are roughly constant throughout the extent of the galaxy. This would not necessarily be the case if the gas that formed those stars had been accreted at different moments and each region had different levels of influence from each inflow.

For all these reasons, we conclude that the SFH of Carina must be dominated by internal evolution. Our interpretation is that, after the first episode formed the majority of the stars in Carina, the physical conditions of the gas made the galaxy unable to form stars for a few gigayears, until it contracted to activate star formation again.

We claim that the temporal gap in star formation in Carina might have been produced by feedback processes. It has been often claimed (e.g., Kaviraj et al. 2007) that feedback can

\footnotetext{
9 For example, relaxation cannot be responsible for the spatial distribution of the different stellar populations in Carina since the relaxation times in dwarf galaxies are much larger than the age of the universe (e.g., Freeman 2008).
}

significantly decrease the efficiency of star formation. In the large halo mass regime (galaxy clusters), this would be due to active galactic nuclei. For low halo masses (dwarf galaxies), this process has been proposed to be caused by supernovae (e.g., Mac Low et al. 1989) and, more recently, stellar-mass black holes (e.g., Leigh et al. 2013).

For example, it has been proposed by simulations (e.g., Revaz et al. 2009) that the star formation of systems with low initial mass $\left(M_{\mathrm{i}}<3 \times 10^{8} M_{\odot}\right)$ could be self-regulated. In these systems, star formation is followed by shocks of energy released by the supernovae that heat and expand the gas. Given that the gas density is relatively low, the gas cooling times are large and can take up to several gigayears to contract to form stars again. If this is the case, the gas available decreases, due to the first episode of star formation, and hence it should contract to a radius smaller than the original to reach the density conditions necessary to reactivate the star formation. This coincides with what we observe in this study, where the second episode occurred preferentially in a region contained within approximately $0.6 \times r_{\text {tidal }}$ of Carina. Unlike the majority of studies about Carina's SFH, we found that the first episode of star formation was the most important one. We also know that the second star-formation episode occurred during a longer period of time. This could explain why, unlike the first episode of star formation, the second one did not halt the star formation and the galaxy could keep forming stars more or less regularly from $\sim 8$ to $\sim 2$ gigayears ago.

But this scenario prompts the question of what is different in Carina to make it have a qualitatively different SFH than any other dSph galaxy? There seems to be a trend in the Local Group, wherein more luminous galaxies (irregular galaxies and brightest classical dwarfs) display more complex SFHs with important components from intermediate-age and young star formation, while dimmer galaxies (dimmest classical dwarfs and ultrafaints) are composed of single old episodes of star formation (e.g., Tolstoy et al. 2009; Brown et al. 2014). Galaxies with larger masses have larger potential wells, and their gas density is generally larger. Given that the cooling of the gas is directly proportional to its density (see details for example on Revaz et al. 2009), these galaxies have shorter cooling times. This enables them to have significant star formation throughout all their history. On the other hand, galaxies with lower masses cannot retain the gas to form stars after the first episode or the gas cannot cool and contract again. Carina has a luminosity similar to the dimmest classical dwarf galaxies, but, unlike them, it has an important component of intermediate-age stars. However, there is evidence that this galaxy may have lost a significant fraction of its mass in the past (Majewski et al. 2000; Muñoz et al. 2006, 2008) and this process is ongoing. This means that in the past Carina could have been significantly brighter than classical dwarfs with currently similar luminosities. Thus, its episodic SFH could represent an intermediate regime between the massive dwarfs with continuous star formation and the least massive dwarfs with only one old episode of star formation. In this context, Carina could have been massive enough to retain the gas after the first episode of star formation, but not massive enough to avoid significant expansion of the gas that stopped the star formation, and given the relatively low gas densities, it could only cool down and contract to form stars again after a couple of gigayears. 


\section{CONCLUSIONS}

In this work we have presented the spatially resolved $\mathrm{SFH}$ (measured in the inner, middle, and outer regions) of the Carina dwarf spheroidal galaxy using deep, wide-field $g$ and $r$ imaging and the MDF from the data of Koch et al. (2006). This is the first time a combination of depth and coverage of this quality is used to derive the SFH of Carina, enabling us to trace its different populations with unprecedented accuracy. The main results of this work can be summarized as follows:

1. The SFH of Carina shows a majority of old metal-poor stars accounting for $54 \pm 4 \%$ of the stellar mass and an intermediate-age population with increasing metallicity with time. The fraction of old stars could be larger considering the possibility that tidal influence from the Milky Way might have preferentially removed old stars from Carina, given that these stars are distributed in a more extended region.

2. Both episodes are separated by a period of no star formation. This temporal gap started $\sim 10$ Gyr ago and stopped $\sim 8$ Gyr ago.

3. Carina displays a positive age radial gradient and a negative metallicity radial gradient. The inner region is dominated by the intermediate-age population, the middle region is composed of similar fractions of old and intermediate-age stars, and the outer region is dominated by the old metal-poor population, with an almost negligible component of intermediate-age stars.

4. The results are consistent with a total stellar mass of $1.60 \pm 0.09 \times 10^{6} M_{\odot}$ within $1.3 \times r_{\text {tidal }}$ and $1.45 \pm$ $0.12 \times 10^{6} M_{\odot}$ within the nominal $r_{\text {tidal }}$. The latter value is larger than previous values derived for Carina. For example, de Boer et al. (2014) derived a value of $1.07 \pm 0.08 \times 10^{6} M_{\odot}$ within the nominal $r_{\text {tidal }}$. We attribute the difference in the masses derived to the increased depth of our photometry, which enabled us to detect more faint, old main-sequence stars.

5. The SFH is consistent with no young ( $<2$ Gyr) stars. We calculated the fraction of blue plume stars in Carina and the fraction of blue stragglers in four other local dwarf spheroidal galaxies. These fractions were calculated using the same mass range (in terms of the mass of the mainsequence turnoff) for both cases, in order to make a meaningful comparison between both fractions. Given that the blue straggler fraction in the local dwarf galaxies is constant and that the blue straggler fraction of Carina is completely consistent with this value, we concluded that the blue plume in this galaxy is consistent with being composed of blue stragglers.

6. The spatially resolved SFH is consistent with being dominated by internal evolution, as opposed to tidal influence from the Milky Way. Our hypothesis is that Carina formed the majority of its stars in a first episode of star formation, then the star formation ceased for a couple of gigayears due to an internal process (e.g., gas heating), and finally, after gas cooling, it reaccreted the gas, producing the second episode of star formation.

F.A.S. acknowledges support from CONICYT Anillo project ACT-1122. R.R.M. acknowledges support from CONICYT through project BASAL PFB-06 and from the FONDECYT project $\mathrm{N}^{\circ} 1120013$. M.G. acknowledges support from the National Science Foundation under award number AST-0908752 and the
Alfred P. Sloan Foundation. S.G.D. was supported in part by the NSF grants AST-1313422, AST-1413600, and AST-1518308. A.E.G. acknowledges support from FONDECYT grant 3150570.

\section{APPENDIX \\ DETERMINING BLUE PLUME AND BLUE STRAGGLER FRACTIONS}

To calculate the contribution of blue stragglers over young stars in the blue plume of Carina, we compare the fraction of blue plume stars in this galaxy with the fraction of blue stragglers in four other local dSph galaxies: Sculptor, Ursa Minor, Draco, and Sextans. We will denominate these the comparison dwarf galaxies, and we use them as a control sample. Since the blue straggler fraction in local dwarf spheroidals has been found to be constant (Momany et al. 2007; Santana et al. 2013), the difference between the blue plume fraction in Carina and the blue straggler fraction in the comparison local dwarf galaxies can be used to estimate the fraction of genuine young stars in Carina, given that there are no young stars in the comparison dwarf galaxies (e.g., Tolstoy et al. 2009, and references therein). To compare the contribution of blue stragglers in Carina with the one in the comparison dwarf galaxies, six important points have to be considered in order to make consistent selection criteria.

1. Carina is composed of two main stellar populations, one old and one at intermediate age. Thus, there are blue straggler stars (BSS) in Carina originating from each of these two stellar populations, whose numbers we denominate as $\mathrm{BSS}_{\mathrm{OLD}}$ and $\mathrm{BSS}_{\mathrm{INT}}$, respectively.

2. A large fraction of $\mathrm{BSS}_{\mathrm{OLD}}$ in Carina cannot be detected in the CMD because their location overlaps with the much more numerous intermediate-age main-sequence stars. Therefore, from the CMD we cannot measure $\mathrm{BSS}_{\mathrm{OLD}}$, and thus for Carina the objective is to calculate $\mathrm{BSS}_{\mathrm{INT}}$.

3. The main-sequence turnoff of the intermediate-age population of Carina is located close to the horizontalbranch stars. Thus, we have to restrict the CMD region where we count the blue plume stars in Carina in order to avoid extremely large contamination in our measurements. Accordingly, the blue plume box defined for Carina includes only the blue stragglers with masses between 1.4 and 1.55 times the mass of the mainsequence turnoff of the intermediate-age population. We denominate this quantity as $\mathrm{BSS}_{\mathrm{INT}, 1.55}$.

4. The blue plume box of Carina so defined is located far from the old main-sequence turnoff, but it still includes a small fraction of blue stragglers that originated from the old population. Therefore, to estimate $\mathrm{BSS}_{\mathrm{INT}, 1.55}$ in Carina, we have to subtract this small contribution.

5. The blue plume and blue straggler numbers measured for all these galaxies have to be normalized to the total number of stars of the stellar population from which the blue stragglers were formed. In this way, we can compare the blue plume star fraction in Carina with the fraction of blue stragglers in the comparison local dwarf galaxies. Because we are using the blue plume box of Carina to determine $\mathrm{BSS}_{\mathrm{INT}}$ (plus the possible contribution of young stars), we normalize the number of blue plume stars by dividing it by the number of stars from the intermediate-age population. For the comparison dwarf 


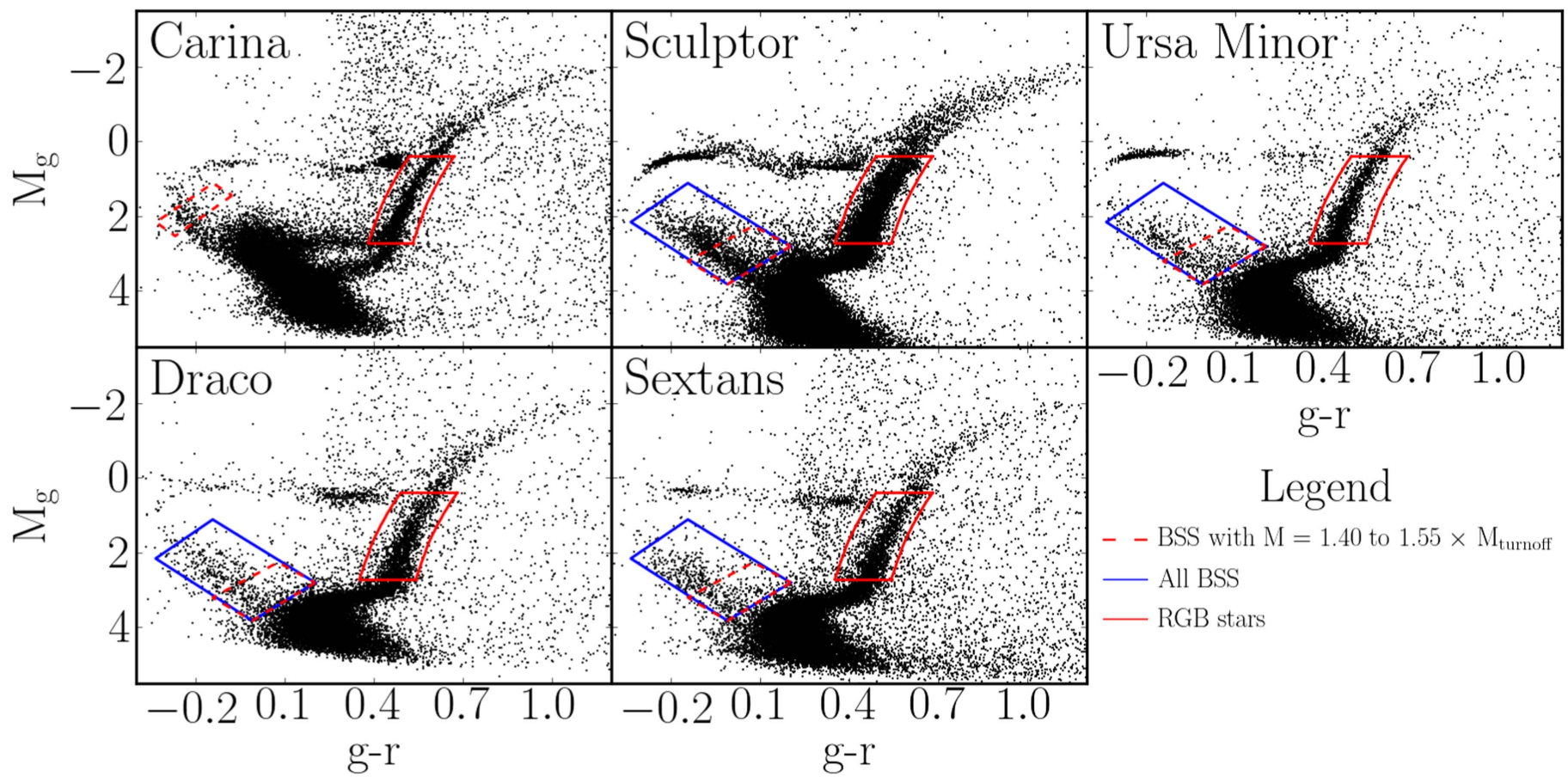

Figure 13. CMDs showing the boxes used to count blue plume stars in Carina blue stragglers in the comparison dwarf galaxies and RGB stars in all of the galaxies. The red solid boxes show the regions where we counted the RGB stars. The red dashed box in the CMD of Carina indicates the blue plume box, which was defined to include stars from 1.40 to 1.55 times the mass of the main-sequence turnoff of the intermediate-age population. The red dashed box in the comparison dwarf galaxies indicates one of the blue straggler boxes used for these galaxies, which was defined to include stars from 1.40 to 1.55 times the mass of the main-sequence turnoff of the old stellar population. The solid blue boxes indicate the second blue straggler box used for the comparison dwarf galaxies, which was defined to count all the blue stragglers in these systems.

galaxies, their CMDs are consistent with being composed of stars that are old ( $12 \mathrm{Gyr})$ and metal-poor. Therefore, the blue stragglers in these systems originate from the old (and only) stellar population.

6. All the types of stars we consider for this analysis (blue plume, blue stragglers, and total stars) are counted in a region that goes from the center up to two times its halflight radius, which corresponds to the spatial coverage of our photometry. This volume is representative of the entire galaxy because in all cases it includes the vast majority of the stars. Nevertheless, some fraction of blue plume or blue straggler stars are not detected simply because they are located outside our coverage. We normalize the populations by dividing by the number of stars located inside two times the half-light radius instead of the total number of stars or the total luminosity of the system.

Taking into account these six considerations, we calculate the blue straggler fraction for the comparison dwarf galaxies as

$$
F_{\mathrm{BSS}, \mathrm{dwarfs}} \equiv \mathrm{BSS}_{\mathrm{TOT}} / N_{\mathrm{TOT}}=\mathrm{BSS}_{\mathrm{OLD}} / N_{\mathrm{OLD}} \text {. }
$$

Because these galaxies are old, there are no intermediate-age blue stragglers in their CMDs. Therefore, they can be counted in the entire magnitude range covering right up from the mainsequence turnoff until just below the horizontal branch. For this reason, $\mathrm{BSS}_{\mathrm{TOT}}$ can be measured directly for the comparison dwarf galaxies. On the other hand, $N_{\text {TOT }}$ cannot be measured directly because a large fraction of the stars are not detected given that their magnitudes are fainter than our detection limit. Therefore, we used the number of RGB stars in these systems as tracers of the total number of stars. We chose them as tracers because they are well above our detection limit and they are not affected by completeness. Moreover, they are very numerous compared to bright stars in other stages of stellar evolution, such as horizontal branch or asymptotic giant branch stars. We note that Santana et al. (2013), using a sample of globular clusters and local dwarf galaxies, demonstrated that the number of RGB stars grows linearly with the luminosity of the system, indicating that there is a simple linear transformation between the number of RGB stars and the total number of stars.

Therefore, we calculate $N_{\text {TOT }}$ as

$$
N_{\mathrm{TOT}}=\mathrm{RGB}_{\mathrm{TOT}} / X_{\mathrm{RGB}, \mathrm{OLD}} \text {, }
$$

in which $\mathrm{RGB}_{\mathrm{TOT}}$ is the total number of RGB stars detected inside the red solid boxes shown in Figure 13. These boxes are defined around an isochrone corresponding to an age of $12 \mathrm{Gyr}$ and an abundance of $[\mathrm{Fe} / \mathrm{H}]$ of -1.9 , which is a good representation of the stellar populations of all these galaxies. The width of the RGB box is 0.095 magnitude at each side of the isochrone, including a magnitude range that goes from 4.9 to 2.4 mag below the RGB tip. Here, $X_{\mathrm{RGB}, \mathrm{OLD}}$ corresponds to the fraction that the selected RGB stars represent from the entire number of stars of an old (age $=12 \mathrm{Gyr}$ ), metal-poor $([\mathrm{Fe} / \mathrm{H}]=-1.9)$ population. To determine this value, we calculate the mass range of the stars in the RGB box and then use a Kroupa IMF (Kroupa 2001) to translate that mass range into a fraction of the stars originally formed. The value obtained for $X_{\mathrm{RGB}, \mathrm{OLD}}$ is $(5.26 \pm 0.34) \times 10^{-4}$. We calculated the uncertainty by repeating the process with different isochrones of slightly different ages and metallicities consistent with the intermediate-age population of the comparison dwarf 
Table 1

Stellar Counts for the Comparison Dwarf Galaxies

\begin{tabular}{|c|c|c|c|c|c|c|c|c|}
\hline Object & $\mathrm{BSS}_{\mathrm{OLD}}{ }^{\mathrm{a}}$ & $\mathrm{BSS}_{\mathrm{OLD}, 1.55^{\mathrm{b}}}$ & $\mathrm{BSS}_{\mathrm{OLD}, \mathrm{BR}}{ }^{\mathrm{c}}$ & $\mathrm{RGB}_{\text {TOT }}$ & $N_{\text {OLD }}{ }^{\mathrm{d}}$ & $F_{\text {BSS }}{ }^{\mathrm{e}}$ & $\frac{\overline{\text { BSSOLD,BR }}}{\text { BSS }_{\mathrm{OLD}}}$ & 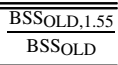 \\
\hline Sculptor & 1434 & 768 & 80 & 4746 & $9.03 \times 10^{6}$ & $(1.59 \pm 0.10) \times 10^{-4}$ & 0.055 & 0.534 \\
\hline Ursa Minor & 567 & 310 & 37 & 1584 & $3.01 \times 10^{6}$ & $(1.88 \pm 0.12) \times 10^{-4}$ & 0.066 & 0.547 \\
\hline Draco & 595 & 330 & 59 & 1697 & $3.23 \times 10^{6}$ & $(1.84 \pm 0.12) \times 10^{-4}$ & 0.100 & 0.554 \\
\hline Sextans & 545 & 258 & 45 & 1564 & $2.98 \times 10^{6}$ & $(1.83 \pm 0.12) \times 10^{-4}$ & 0.083 & 0.474 \\
\hline Average & & & & & & $1.79 \times 10^{-4}$ & 0.076 & 0.528 \\
\hline Standard Deviation & & & & & & $1.34 \times 10^{-5}$ & 0.020 & 0.037 \\
\hline
\end{tabular}

Notes.

${ }^{\mathrm{a}}$ BSS from the old population.

b BSS from the old population with $M=(1.4-1.55) \times M_{\text {turnoff }}$.

${ }^{c}$ BSS from the old population bright enough to fall in the blue plume box defined for Carina.

d Number of stars from the old stellar population.

${ }^{\mathrm{e}}$ Fraction of blue stragglers as measured from Equation (1).

galaxies, and by taking the uncertainty as the standard deviation of the different values obtained for $X_{\mathrm{RGB}, \mathrm{OLD}}$. Replacing Equation (2) into Equation (1) we obtain

$$
F_{\mathrm{BSS}, \mathrm{dwarfs}}=X_{\mathrm{RGB}, \mathrm{OLD}} \times \frac{\mathrm{BSS}_{\mathrm{TOT}}}{\mathrm{RGB}_{\mathrm{TOT}}},
$$

whose values are given in the seventh column of Table 1.

For the case of Carina, the blue plume includes, in principle, both blue stragglers and young stars. Therefore, the number of blue plume stars measured in Carina is given by

$$
N_{\mathrm{BP}, \mathrm{Car}}=\mathrm{BSS}_{\mathrm{INT}, \mathrm{BOX}}+\mathrm{BSS}_{\mathrm{OLD}, \mathrm{BOX}}+Y S_{\mathrm{BOX}} \text {, }
$$

where the BOX subscript refers to the CMD location indicated in Figure 13 (red dashed box). In Equation (4), $Y S_{\mathrm{BOX}}$ represents the number of young stars falling in the blue plume box. Based on the magnitude limits of the blue plume box defined for Carina, we calculate that the blue stragglers from the intermediate-age population included in the box have masses ranging from 1.40 to 1.55 times the mass of the intermediate-age population main-sequence turnoff of Carina. We estimate this mass range by translating the magnitude limits of the blue plume box of Carina into stellar masses based on a very young $($ age $=1 \mathrm{~Gy}$ ) isochrone with the metallicity of the intermediate-age population of Carina $([\mathrm{Fe} / \mathrm{H}]=-1.4)$. Given that $\mathrm{BSS}_{\mathrm{INT}, \mathrm{BOX}}$ only includes the stars in that mass range, we will denominate this quantity as $\mathrm{BSS}_{\mathrm{INT}, 1.55}$. Analogously, BSS $_{\mathrm{OLD}, \mathrm{BOX}}$ only includes the brightest blue stragglers that come from the old population of Carina, and thus we denominate this quantity as $\mathrm{BSS}_{\mathrm{OLD}, \mathrm{BR}}$. Therefore, Equation (4) can be rewritten as

$$
N_{\mathrm{BP}, \mathrm{Car}}=\mathrm{BSS}_{\mathrm{INT}, 1.55}+\mathrm{BSS}_{\mathrm{OLD}, \mathrm{BR}}+Y S_{\mathrm{BOX}} \text {. }
$$

The final goal of this analysis is to compare the blue plume fraction of Carina with the blue straggler fraction of the comparison dwarf galaxies. All the stars in the comparison dwarf galaxies are old and metal-poor, and unlike Carina, their blue stragglers come from a single stellar population. For this reason, we subtract the contribution from the old blue stragglers to the blue plume counts in Carina. In this way, we can compare the blue stragglers from a single population in Carina with those of the comparison dwarf galaxies. Estimating BSS $_{\mathrm{OLD}, \mathrm{BR}}$ entails calculating the fraction of blue stragglers from the old population falling in the blue plume box defined for Carina. We denominate this quantity as $X_{\mathrm{BR}, \mathrm{TOT}}$, and it is defined as

$$
X_{\mathrm{BR}, \mathrm{TOT}} \equiv \frac{\mathrm{BSS}_{\mathrm{OLD}, \mathrm{BR}}}{\mathrm{BSS}_{\mathrm{OLD}}} .
$$

To estimate this quantity, we can use the measurements directly from Table 1. Given that $X_{\mathrm{BR}, \mathrm{TOT}}$ is fairly constant in the comparison dwarf galaxies, for Carina we estimate it as the average of the values found for the comparison dwarf galaxies and its uncertainty as the standard deviation. In this way, we obtain

$$
X_{\mathrm{BR}, \mathrm{TOT}}=0.076 \pm 0.020 \text {, }
$$

which implies

$$
\mathrm{BSS}_{\mathrm{OLD}, \mathrm{BR}}=X_{\mathrm{BR}, \mathrm{TOT}} \times \frac{\mathrm{BSS}_{\mathrm{OLD}}}{N_{\mathrm{OLD}}} \times N_{\mathrm{OLD}} .
$$

Table 1 also shows the fractions of old blue stragglers over the total number of old stars for the comparison dwarf galaxies. This value is almost constant in these galaxies, with a standard deviation that represents only $\sim 7 \%$ of the average. Moreover, Santana et al. (2013) found that the fraction of blue stragglers in local dwarf galaxies is practically constant. For these reasons, we estimate $\frac{\mathrm{BSS}_{\text {OLD }}}{N_{\text {OLD }}}$ for Carina as the average value found for dwarf galaxies with the uncertainty equal to the standard deviation:

$$
\frac{\mathrm{BSS}_{\mathrm{OLD}}}{N_{\mathrm{OLD}}}=(1.78 \pm 0.13) \times 10^{-4},
$$

which implies that (see Equation (8))

$$
\mathrm{BSS}_{\mathrm{OLD}, \mathrm{BR}}=(1.36 \pm 0.19) \times 10^{-5} \times N_{\mathrm{OLD}}
$$

To calculate $N_{\mathrm{OLD}}$, we estimate it in an analogous way as $N_{\text {TOT }}$ for the comparison dwarf galaxies, that is, by using the RGB stars as tracers of the total number of stars formed in the corresponding star-formation episode. The RGB box defined for Carina (red continuous box of Figure 13) includes RGB stars that belong to the old population $\left(\mathrm{RGB}_{\mathrm{OLD}}\right)$ and $\mathrm{RGB}$ stars that belong to the intermediate-age population $\left(\mathrm{RGB}_{\mathrm{INT}}\right)$. By using the results obtained from the SFH analysis performed on Carina, we calculate that $42 \%$ of the stars in the RGB box of Carina belong to the old population. Additionally, as we pointed 
out earlier, $X_{\mathrm{RGB}, \mathrm{OLD}}=(5.26 \pm 0.34) \times 10^{-4}$, so we obtain

$$
N_{\mathrm{OLD}}=0.42 \frac{\times \mathrm{RGB}_{\mathrm{TOT}}}{X_{\mathrm{RGB}, \mathrm{OLD}}}=(799 \pm 52) \times \mathrm{RGB}_{\mathrm{TOT}},
$$

and using Equation (10), we obtain

$$
\mathrm{BSS}_{\mathrm{OLD}, \mathrm{BR}}=(0.011 \pm 0.002) \times \mathrm{RGB}_{\mathrm{TOT}} .
$$

By subtracting this equation from Equation (5) and reordering, we get

$$
\begin{aligned}
& N_{\mathrm{BP}, \mathrm{Car}}-0.011 \times \mathrm{RGB}_{\mathrm{TOT}} \\
& \quad=\mathrm{BSS}_{\mathrm{INT}, 1.55}\left(1+\frac{Y S_{\mathrm{BOX}}}{\mathrm{BSS}_{\mathrm{INT}, 1.55}}\right) .
\end{aligned}
$$

The next step is to estimate the total number of blue stragglers in Carina that come from the intermediate-age population $\left(\mathrm{BSS}_{\mathrm{INT}}\right)$ based on the value of $\mathrm{BSS}_{\mathrm{INT}, 1.55}$. For this we will assume that the fraction of blue stragglers in the mass range of 1.4 to 1.55 times the mass of the main-sequence turnoff over the total number of blue stragglers of that particular population is the same for the old and intermediate-age populations:

$$
\frac{\mathrm{BSS}_{\mathrm{INT}, 1.55}}{\mathrm{BSS}_{\mathrm{INT}}}=\frac{\mathrm{BSS}_{\mathrm{OLD}, 1.55}}{\mathrm{BSS}_{\mathrm{OLD}}} \text {. }
$$

This assumption is based on two observations. The first one is that there is no significant dependence of the fraction of blue stragglers on the age of the stellar population for ages larger than 3-4 Gyr (de Marchi et al. 2006; Ahumada \& Lapasset 2007; Xin et al. 2007). The second one is that the fraction $\frac{\mathrm{BSS}_{\mathrm{OLD}, 1.55}}{\text { BSS }_{\text {OLD }}}$ is practically constant among the comparison dwarf galaxies, displaying a standard deviation that represents only $7 \%$ of the average value. By calculating $\frac{\text { BSS }_{\text {OLD } 1.55}}{\text { BSS }_{\text {OLD }}}$ for the comparison dwarf galaxies, we obtain

$$
\frac{\mathrm{BSS}_{\mathrm{OLD}, 1.55}}{\mathrm{BSS}_{\mathrm{OLD}}}=0.528 \pm 0.037
$$

which implies

$$
\text { BSS }_{\mathrm{INT}, 1.55}=0.528 \times \mathrm{BSS}_{\mathrm{INT}} .
$$

Therefore

$$
\begin{gathered}
1.894\left(N_{\mathrm{BP}, \mathrm{Car}}-0.011 \times \mathrm{RGB}_{\mathrm{TOT}}\right) \\
=\mathrm{BSS}_{\mathrm{INT}}\left(1+\frac{Y S_{\mathrm{BOX}}}{\mathrm{BSS}_{\mathrm{INT}, 1.55}}\right) .
\end{gathered}
$$

Now to calculate the blue plume fraction of Carina, $F_{\mathrm{BP}, \mathrm{Car}}$, we have to divide Equation (17) by the total number of stars from the intermediate-age population, $N_{\text {INT }}$. By doing this, we obtain

$$
\begin{aligned}
F_{\mathrm{BP}, \mathrm{Car}} & =\frac{1.894\left(N_{\mathrm{BP}, \mathrm{Car}}-0.011 \times \mathrm{RGB}_{\mathrm{TOT}}\right)}{N_{\mathrm{INT}}} \\
& =F_{\mathrm{BSS}, \mathrm{Car}}\left(1+\frac{Y S_{\mathrm{BOX}}}{\mathrm{BSS}_{\mathrm{INT}, 1.55}}\right),
\end{aligned}
$$

where $F_{\mathrm{BSS}, \mathrm{Car}}$ is the fraction of genuine blue stragglers in Carina, the quantity that has been consistently calculated to be compared to the fraction of blue stragglers in the comparison dwarf galaxies $\left(F_{\mathrm{BSS}, \mathrm{dwarf}}\right)$. Now the only thing remaining is to calculate $N_{\text {INT }}$, which we will do in the same way as for $N_{\text {OLD }}$, by using the number of RGB stars as a tracer of the total number of stars. Analogous to our definition of $N_{\mathrm{OLD}}$ in Equation (2), we define $N_{\text {INT }}$ as

$$
N_{\mathrm{INT}}=\mathrm{RGB}_{\mathrm{INT}} / X_{\mathrm{RGB}, \mathrm{INT}} \text {, }
$$

where $X_{\mathrm{RGB}, \mathrm{OLD}}$ corresponds to the fraction $\mathrm{RGB}_{\mathrm{INT}}$ from the entire intermediate-age population. To model the intermediate-age population of Carina, we will use a Dartmouth (Dotter et al. 2008) isochrone with an age of $6 \mathrm{Gyr}$ and an abundance of $[\mathrm{Fe} / \mathrm{H}]=-$ 1.4 , which produces an accurate fit to the CMD location of the intermediate-age population of Carina. As we did for $X_{\mathrm{RGB}, \mathrm{OLD}}$, we determine $X_{\mathrm{RGB}, \mathrm{INT}}$ by translating the magnitude limits of the RGB box into a stellar mass range, according to the isochrone representative of the population. Then, using a Kroupa IMF (Kroupa 2001), we translate that mass range into a fraction of the stars originally formed for the intermediate-age population. By doing this, we obtain a value of $(8.68 \pm 0.39) \times 10^{4}$ for $X_{\text {RGB,INT }}$. By using the results obtained from the SFH analysis performed on Carina, we calculate that $58 \%$ of the stars in the RGB box of Carina belong to the intermediate-age population. Thus, by replacing these values into Equation (19), we obtain

$$
N_{\mathrm{INT}}=\frac{0.58 \times \mathrm{RGB}_{\mathrm{TOT}}}{8.68 \times 10^{-4}}=668 \times \mathrm{RGB}_{\mathrm{TOT}} .
$$

Then, by replacing this equation into Equation (18), we can finally obtain

$$
\begin{aligned}
F_{\mathrm{BP}, \mathrm{Car}} & =2.84 \times 10^{-3} \times \frac{N_{\mathrm{BP}, \mathrm{Carina}}}{N_{\mathrm{RGB}}}-3.08 \times 10^{-5} \\
& =F_{\mathrm{BSS}, \mathrm{Car}}\left(1+\frac{Y S_{\mathrm{BOX}}}{\mathrm{BSS}_{\mathrm{INT}, 1.55}}\right) .
\end{aligned}
$$

This allows us to calculate $F_{\mathrm{BP}, \text { Car }}$ directly from the observables $N_{\mathrm{BP}, \mathrm{Car}}$ and $N_{\mathrm{RGB}}$. These values correspond respectively to the number of stars in the dashed and solid red lines on Carina's CMD in Figure 13. The right-hand side of the equation depends on the fraction $\frac{Y S_{\mathrm{BOX}}}{\mathrm{BSS}_{\mathrm{INT}, 1.55}}$. This quantity is the ratio of genuine young stars over the genuine blue stragglers that are located in the blue plume box of Carina, so it can significantly help us in elucidating the nature of the stars in this CMD region. The blue plume fraction of Carina is then the genuine blue straggler fraction multiplied by a correction factor that depends only on the fraction of young stars in the blue plume box. By replacing the observed number of stars in the different CMD boxes (listed in Tables 1 and 2) in Equations (3) and (21), we obtain the values for the blue plume fraction for Carina $\left(F_{\mathrm{BP}, \mathrm{Car}}\right)$ and the average blue straggler fraction for the comparison dwarf galaxies $\left(\overline{F_{\mathrm{BSS}, \mathrm{dwarfs}}}\right)$ :

$$
\begin{aligned}
F_{\mathrm{BP}, \mathrm{Car}} & =F_{\mathrm{BSS}, \mathrm{Car}}\left(1+\frac{Y S_{\mathrm{BOX}}}{\mathrm{BSS}_{\mathrm{INT}, 1.55}}\right) \\
& =(1.89 \pm 0.25) \times 10^{-4},
\end{aligned}
$$

and

$$
\overline{F_{\mathrm{BSS}, \mathrm{dwarfs}}}=(1.79 \pm 0.13) \times 10^{-4},
$$

where the error cited for $\overline{F_{\mathrm{BSS}, \text { dwarfs }}}$ corresponds to the standard deviation calculated for the comparison dwarf galaxies. If we compare these last two equations, we can see that the blue 
Table 2

Stellar Counts for Carina

\begin{tabular}{|c|c|c|c|c|c|c|c|c|c|c|}
\hline $\mathrm{RGB}_{\text {TOT }}{ }^{\mathrm{a}}$ & RGB $_{\text {INT }}{ }^{b}$ & $\mathrm{RGB}_{\mathrm{OLD}}{ }^{\mathrm{c}}$ & $N_{\text {INT }}{ }^{\mathrm{d}}$ & $N_{\text {OLD }}{ }^{\mathrm{e}}$ & $\mathrm{BSS}_{\mathrm{OLD}}{ }^{\mathrm{f}}$ & $\overline{\mathrm{BSS}_{\mathrm{OLD}, \mathrm{BR}}{ }^{\mathrm{g}}}$ & $\mathrm{BSSS}_{\mathrm{TOT}, 1.55^{\mathrm{h}}}$ & $\mathrm{CBSS}_{\mathrm{INT}, 1.55^{\mathrm{i}}}$ & $\overline{B S S S}_{\text {INT }^{j}}{ }^{\mathrm{j}}$ & $F_{\mathrm{BP}}{ }^{\mathrm{k}}$ \\
\hline 1724 & 1000 & 725 & $1.15 \times 10^{6}$ & $1.38 \times 10^{6}$ & 246 & 19 & 134 & 115 & 218 & $(1.89 \pm 0.25) \times 10^{-4}$ \\
\hline
\end{tabular}

Notes.

${ }^{a}$ Total number of stars falling in the RGB box defined for Carina.

${ }^{\mathrm{b}}$ RGB stars from the intermediate-age population.

${ }^{c}$ RGB stars from the old population.

${ }^{\mathrm{d}}$ Number of stars from the old stellar population.

${ }^{\mathrm{e}}$ Number of stars from the intermediate-age population.

${ }^{\mathrm{f}}$ BSS from the old population.

${ }^{g}$ BSS from the old population bright enough to fall in the blue plume box.

${ }^{\mathrm{h}}$ BSSs that fall in the blue plume box of Carina.

${ }^{\mathrm{i}}$ BSSs that form the intermediate-age population with $M=(1.4-1.55) \times M_{\text {turnoff. }}$.

${ }^{j}$ BSS from the intermediate-age population.

${ }^{\mathrm{k}}$ Fraction of blue stragglers as measured from Equation (21).

plume fraction of Carina is consistent within the errors with the blue straggler fraction in the comparison dwarf galaxies, or equivalently, $Y S_{\mathrm{BOX}}$ is consistent with being equal to zero. Based on this result, we conclude that there are no genuine young (age $<2 \mathrm{Gyr}$ ) stars in Carina, and that the stars in the blue plume of this galaxy are instead blue stragglers. This is illustrated in Figure 12, where we plot the blue straggler fractions of all the comparison dwarf galaxies and the blue plume fraction of Carina. Based on our calculations, we derive that the maximum fraction of young stars in the blue plume of Carina consistent with our uncertainties is $16.5 \%$.

According to our original SFH derivation for Carina, we calculated that $(1.45 \pm 0.19) \%$ of the stars in Carina are younger than 2 Gyr. Thus, if $16.5 \%$ of those young stars were authentic young stars as opposed to blue stragglers, then only $0.24 \%$ of the stars would be younger than $2 \mathrm{Gyr}$. This fraction is practically negligible, and given that the highest young star fraction that is consistent with our measurements is comparable to the error in the SFH derivation, we confirm our previous conclusion that there are no authentic young stars in Carina and that its blue plume is composed of blue stragglers.

\section{REFERENCES}

Ahumada, J. A., \& Lapasset, E. 2007, A\&A, 463, 789

Aparicio, A., \& Hidalgo, S. L. 2009, AJ, 138, 558

Armandroff, T. E., \& Da Costa, G. S. 1991, AJ, 101, 1329

Battaglia, G., Irwin, M., Tolstoy, E., de Boer, T., \& Mateo, M. 2012, ApJL, 761, L31

Bono, G., Stetson, P. B., Walker, A. R., et al. 2010, PASP, 122, 651

Brown, T. M., Tumlinson, J., Geha, M., et al. 2014, ApJ, 796, 91

Cannon, R. D., Hawarden, T. G., \& Tritton, S. B. 1977, MNRAS, 180, 81

Carretta, E., \& Gratton, R. G. 1997, A\&AS, 121, 95

Coppola, G., Stetson, P. B., Marconi, M., et al. 2013, ApJ, 775, 6

de Boer, T. J. L., Tolstoy, E., Hill, V., et al. 2012, A\&A, 539, A103

de Boer, T. J. L., Tolstoy, E., Lemasle, B., et al. 2014, A\&A, 572, A10

de Marchi, F., de Angeli, F., Piotto, G., Carraro, G., \& Davies, M. B. 2006 ,

A\&A, 459, 489

Dotter, A., Chaboyer, B., Jevremović, D., et al. 2008, ApJS, 178, 89

El-Badry, K., Wetzel, A. R., Geha, M., et al. 2015, ApJ, 820, 131

Freeman, K. C. 2008, Msngr, 134, 28

Geha, M., Brown, T. M., Tumlinson, J., et al. 2013, ApJ, 771, 29

Gosnell, N. M., Mathieu, R. D., Geller, A. M., et al. 2014, ApJL, 783, L8

Gratton, R. G., Carretta, E., \& Bragaglia, A. 2012, A\&ARv, 20, 50

Grebel, E. K. 1999, in IAU Symp. 192, The Stellar Content of Local Group Galaxies, ed. P. Whitelock \& R. Cannon (San Francisco, CA: ASP), 17

Helmi, A., Irwin, M. J., Tolstoy, E., et al. 2006, ApJL, 651, L121

Hurley-Keller, D., Mateo, M., \& Nemec, J. 1998, AJ, 115, 1840
Irwin, M., \& Hatzidimitriou, D. 1995, MNRAS, 277, 1354

Karczmarek, P., Pietrzyński, G., Gieren, W., et al. 2015, AJ, 150, 90

Kauffmann, G., White, S. D. M., \& Guiderdoni, B. 1993, MNRAS, 264, 201

Kaviraj, S., Kirkby, L. A., Silk, J., \& Sarzi, M. 2007, MNRAS, 382, 960

Koch, A., Grebel, E. K., Wyse, R. F. G., et al. 2006, AJ, 131, 895

Kouwenhoven, M. B. N., Brown, A. G. A., Goodwin, S. P., Portegies Zwart, S. F., \& Kaper, L. 2009, A\&A, 493, 979

Kroupa, P. 2001, MNRAS, 322, 231

Larson, R. B. 2002, MNRAS, 332, 155

Leigh, N. W. C., Böker, T., Maccarone, T. J., \& Perets, H. B. 2013, MNRAS, 429, 2997

Lemasle, B., Hill, V., Tolstoy, E., et al. 2012, A\&A, 538, A100

Mac Low, M.-M., McCray, R., \& Norman, M. L. 1989, ApJ, 337, 141

Majewski, S. R., Ostheimer, J. C., Patterson, R. J., et al. 2000, AJ, 119, 760

Mateo, M., Hurley-Keller, D., \& Nemec, J. 1998, AJ, 115, 1856

Mateo, M. L. 1998, ARA\&A, 36, 435

McConnachie, A. W. 2012, AJ, 144, 4

McConnachie, A. W., \& Côté, P. 2010, ApJL, 722, L209

McMonigal, B., Bate, N. F., Lewis, G. F., et al. 2014, MNRAS, 444, 3139

Momany, Y., Held, E. V., Saviane, I., et al. 2007, A\&A, 468, 973

Monelli, M., Pulone, L., Corsi, C. E., et al. 2003, AJ, 126, 218

Muñoz, R. R., Geha, M., \& Willman, B. 2010, AJ, 140, 138

Muñoz, R. R., Majewski, S. R., \& Johnston, K. V. 2008, ApJ, 679, 346

Muñoz, R. R., Majewski, S. R., Zaggia, S., et al. 2006, ApJ, 649, 201

Nonino, M., Bertin, E., da Costa, L., et al. 1999, A\&AS, 137, 51

Pasetto, S., Grebel, E. K., Berczik, P., Chiosi, C., \& Spurzem, R. 2011, A\&A, 525, A99

Piatek, S., Pryor, C., Olszewski, E. W., et al. 2003, AJ, 126, 2346

Pietrinferni, A., Cassisi, S., Salaris, M., \& Castelli, F. 2004, ApJ, 612, 168

Pilkington, K., \& Gibson, B. K. 2012, in Nuclei in the Cosmos (Trieste, SISSA), 227

Revaz, Y., Jablonka, P., Sawala, T., et al. 2009, A\&A, 501, 189

Rizzi, L., Held, E. V., Bertelli, G., \& Saviane, I. 2003, ApJL, 589, L85

Saha, A., Monet, D. G., \& Seitzer, P. 1986, AJ, 92, 302

Sales, L. V., Helmi, A., \& Battaglia, G. 2010, AdAst, 2010, 18

Santana, F. A., Muñoz, R. R., Geha, M., et al. 2013, ApJ, 774, 106

Schlegel, D. J., Finkbeiner, D. P., \& Davis, M. 1998, ApJ, 500, 525

Smecker-Hane, T. A., Stetson, P. B., Hesser, J. E., \& Vandenberg, D. A 1996, in ASP Conf. Ser. 98, From Stars to Galaxies: the Impact of Stellar Physics on Galaxy Evolution, ed. C. Leitherer, U. Fritze-von-Alvensleben, \& J. Huchra (San Francisco, CA: ASP), 328

Spada, F., Demarque, P., Kim, Y.-C., \& Sills, A. 2013, ApJ, 776, 87

Starkenburg, E., Hill, V., Tolstoy, E., et al. 2010, A\&A, 513, A34

Stetson, P. B. 1994, PASP, 106, 250

Tolstoy, E., Hill, V., \& Tosi, M. 2009, ARA\&A, 47, 371

Unavane, M., Wyse, R. F. G., \& Gilmore, G. 1996, MNRAS, 278, 727

VandenBerg, D. A., Stetson, P. B., \& Brown, T. M. 2015, ApJ, 805, 103

Walker, M. G., Mateo, M., Olszewski, E. W., et al. 2009, ApJ, 704, 1274

Weisz, D. R., Dolphin, A. E., Skillman, E. D., et al. 2014a, ApJ, 789, 147

Weisz, D. R., Dolphin, A. E., Skillman, E. D., et al. 2014b, ApJ, 789, 148

Xin, Y., Deng, L., \& Han, Z. W. 2007, ApJ, 660, 319

York, D., Adelman, J., Anderson, J. E., Jr., et al. 2000, AJ, 120, 1579

Zinn, R., \& West, M. J. 1984, ApJS, 55, 45 